\title{
Numerical Approximation of Oscillatory Solutions of Hyperbolic-Elliptic Systems of Conservation Laws by Multiresolution Schemes
}

\author{
Stefan Berres ${ }^{1}$, Raimund Bürger ${ }^{2}, *$ and Alice Kozakevicius ${ }^{3}$ \\ ${ }^{1}$ Departamento de Ciencias Matemáticas y Físicas, Universidad Católica de Temuco, \\ Temuco, Chile \\ ${ }^{2} \mathrm{CI}^{2} \mathrm{MA}$ and Departamento de Ingeniería Matemática, Facultad de Ciencias Físicas \\ y Matemáticas, Universidad de Concepción, Casilla 160-C, Concepción, Chile \\ ${ }^{3}$ Departamento de Matemática-CCNE, Universidade Federal de Santa Maria, \\ Av. Roraima, 1000, Campus Universitário, Santa Maria, RS, CEP 97105-900, Brazil
}

Received 23 April 2009; Accepted (in revised version) 26 June 2009

Available online 30 July 2009

\begin{abstract}
The generic structure of solutions of initial value problems of hyperbolic-elliptic systems, also called mixed systems, of conservation laws is not yet fully understood. One reason for the absence of a core well-posedness theory for these equations is the sensitivity of their solutions to the structure of a parabolic regularization when attempting to single out an admissible solution by the vanishing viscosity approach. There is, however, theoretical and numerical evidence for the appearance of solutions that exhibit persistent oscillations, so-called oscillatory waves, which are (in general, measure-valued) solutions that emerge from Riemann data or slightly perturbed constant data chosen from the interior of the elliptic region. To capture these solutions, usually a fine computational grid is required. In this work, a version of the multiresolution method applied to a WENO scheme for systems of conservation laws is proposed as a simulation tool for the efficient computation of solutions of oscillatory wave type. The hyperbolic-elliptic $2 \times 2$ systems of conservation laws considered are a prototype system for three-phase flow in porous media and a system modeling the separation of a heavy-buoyant bidisperse suspension. In the latter case, varying one scalar parameter produces elliptic regions of different shapes and numbers of points of tangency with the borders of the phase space, giving rise to different kinds of oscillation waves.
\end{abstract}

AMS subject classifications: 76T20, 35L65, 65M06, 76M20, 35M10, 35R25

Key words: Hyperbolic-elliptic system, conservation law, oscillation wave, numerical simulation, multiresolution method, sedimentation model.

*Corresponding author.

URL: http: //www.ing-mat.udec.cl/ rburger/

Email: sberres@uct.cl (S. Berres),rburger@ing-mat.udec.cl (R. Bürger), alicek@smail.ufsm.br (A. Kozakevicius)

http:/ / www.global-sci.org/aamm 


\section{Introduction}

\subsection{Scope of the paper}

We consider first-order systems of two scalar, nonlinear, strongly coupled conservation laws

$$
\partial_{t} \phi_{i}+\partial_{x} f_{i}\left(\phi_{1}, \phi_{2}\right)=0, \quad i=1,2, \quad x \in \mathbb{R}, \quad t>0,
$$

subject to the initial condition

$$
\phi_{i}(x, 0)=\phi_{i, 0}(x), \quad i=1,2, \quad x \in \mathbb{R} .
$$

We recall that the system (1.1) is called hyperbolic at a point $\left(\phi_{1}, \phi_{2}\right)$ if the Jacobian $\mathcal{J}_{f}$ of the flux vector

$$
f=\left(f_{1}, f_{2}\right)^{\mathrm{T}}
$$

evaluated at $\left(\phi_{1}, \phi_{2}\right)$,

$$
\mathcal{J}_{f}\left(\phi_{1}, \phi_{2}\right):=\left(J_{i j}\left(\phi_{1}, \phi_{2}\right)\right)_{i, j=1,2}:=\left(\frac{\partial f_{i}}{\partial \phi_{j}}\left(\phi_{1}, \phi_{2}\right)\right)_{i, j=1,2},
$$

has real eigenvalues, that is, if the discriminant

$$
\Delta\left(\phi_{1}, \phi_{2}\right):=\left(\left(J_{11}-J_{22}\right)^{2}+4 J_{12} J_{21}\right)\left(\phi_{1}, \phi_{2}\right)
$$

is non-negative, and strictly hyperbolic if these eigenvalues are moreover distinct, that is, if $\Delta\left(\phi_{1}, \phi_{2}\right)>0$. If $\Delta\left(\phi_{1}, \phi_{2}\right)<0$, then $\mathcal{J}_{f}\left(\phi_{1}, \phi_{2}\right)$ has a pair of complex conjugate eigenvalues and (1.1) is called elliptic at that point. The set of all points $\left(\phi_{1}, \phi_{2}\right)$ at which (1.1) is elliptic is called elliptic region.

It is the purpose of this contribution to study numerically the solution behaviour of (1.1), (1.2) when the initial data are chosen as a pair of constants from the elliptic region, and where these constants are slightly perturbed on a small interval (corresponding to a small number of cells of a spatial discretization). For this setting, and a particular system, Frid and Liu [22] observed highly oscillatory but strongly localized solutions, which they termed oscillation waves. We herein capture, and in part analyze, such oscillations first for the system studied in [22] (Model 1), and then for a hyperbolic-elliptic system that emerges from a model of sedimentation of a bidisperse suspension (Model 2) [6,11]. The novelty of our approach is that we employ a multiresolution (MR) method, which adaptively concentrates computational effort associated with a given numerical scheme for systems of conservation laws on areas of strong variation of the solution. In our case, the method can be advantageously employed to capture the oscillations due to the mixed-type nature of the system. 


\subsection{Mixed-type systems of conservation laws}

Our interest in mixed-type or hyperbolic-elliptic systems of conservation laws originated in models of sedimentation of polydisperse suspensions. In this application, the governing equations are a system of $N$ scalar, nonlinear, strongly coupled conservation laws for the general case of a suspension with particles of $N$ species differing in size or density. If particle species differ in density, then the phase space of admissible concentration vectors contains regions where the system is non-hyperbolic (elliptic for $N=2$ ). See [11] for the analysis of some specific models; our treatment will be limited to the case $N=2$. Other applications of mixed-type systems include transonic flow, traffic flow, one-dimensional unsteady flow of a van der Waals gas, propagation of phase boundaries in elastic bars, and multiphase flow (e.g., water, gas, oil) in porous media. Of particular interest are systems of conservation laws modeling three-phase flow in porous media, since there are some similarities to systems modeling polydisperse sedimentation.

In fact, models for multiphase flow in porous media formed the main stimulus for intense research related to systems that change type. We refer to $[19,20,30,31,40,41]$ for overviews of the theory of mixed systems of conservation laws and their applications; a concise review based on these papers is provided in [11, Sect. 6.2]. In practical numerical calculations, the existence of elliptic regions usually does not compel computational instabilities, which is why the change of type was not noticed at first; for example, some of the numerical calculations performed in [8] for various systems of conservation laws modeling polydisperse sedimentation include the hyperbolicelliptic case, but oscillations were not observed. For the latter model, constructions of solutions by the front tracking method for the hyperbolic-elliptic case corresponding to a bidisperse suspension with particles having different sizes and densities are made in [4]. The examples in [4] start from "hyperbolic" initial data, and it turns out that the solution not only avoids the elliptic region, but presents some features that do not appear in the strictly hyperbolic case, including the formation of a continuously varying steady-state solution due to the presence of an umbilic point in the hyperbolic subregion. One reason for the lack of oscillations is, of course, the numerical diffusion introduced by most numerical schemes, which turns the solution of the system of conservation laws into a solution that is similar to the exact solution of a well-posed parabolic system.

Nevertheless, the existence of oscillatory solutions (which are measure-valued solutions) for non-hyperbolic systems has been proved both analytically and numerically [21]. In fact, using a first-order finite difference scheme, Frid and Liu [22] showed that approximate solutions to Riemann problems with initial data inside elliptic regions may present persistent large-amplitude oscillation waves. Numerical studies have also shown that when one state belongs to the hyperbolic region and the other to the elliptic, the solution is acceptable, but still displays some oscillations. On the other hand, when both states are located in the hyperbolic region, the numerical solutions seem to be stable. It has furthermore been demonstrated that solutions to Riemann 
problems with "hyperbolic" initial data avoid the elliptic regions. The distinction between Riemann and more general Cauchy problems is essential, since this avoidance of the elliptic region is not true for more general Cauchy data [27,45].

In general, the well-posedness theory for mixed systems of conservation laws is not yet as developed as the theory of hyperbolic systems of conservation laws. In the latter case, it is known that admissibility criteria or entropy conditions are required to make the model well-posed. One of these criteria can be derived by adding a diffusion term to the right-hand side of (1.1), and then to study the traveling waves of the resulting convection-diffusion system when the regularizing diffusion parameter tends to zero. On the other hand, to regard a mixed-type system as an inviscid limit of a parabolic system is not entirely satisfactory since the stability or admissibility of such shock waves may depend on the form of the regularizing (nonlinear) diffusion matrices. Papers that discuss these or related issues include $[1,3,39,40]$. In particular, it is not clear what the physical diffusion matrix for polydisperse sedimentation models (our Model 2) should look like. This problem can possibly be resolved with the help of computational and experimental studies of hydrodynamic diffusion [16,34,49].

Summarizing, we can say that although the mathematical and numerical theory as well as the general understanding of mixed systems have advanced significantly recently, most essential problems have remained unsolved and there exists no core theory and there is no general agreement on admissibility criteria for mixed systems.

\subsection{High-resolution and multiresolution schemes}

High-resolution shock-capturing schemes are a well-established tool for the accurate numerical solution of systems of conservation laws. Methods of this type include essentially non-oscillatory (ENO) schemes [46], weighted essentially non-oscillatory (WENO) schemes (see [47]), and non-oscillatory central schemes [33, 44]; see also $[36,48]$. Clearly, resolution is gained by these methods at increased computational cost, for example when reconstructing the solution by higher order polynomials. The multiresolution (MR) method has been devised (at least, originally) to reduce the computational cost of high-resolution methods. The solution of a system of conservation laws usually exhibits strong variations (shocks) in small regions but behaves smoothly elsewhere. The MR technique adaptively concentrates computational effort on the regions of strong variation. It goes back to Harten [25] for hyperbolic equations. See Chiavassa et al. [15] and Müller [43] for a recent review on MR methods for hyperbolic conservation laws.

The MR method used herein, which is adapted from [12,32], is based on grid refinement to resolve gradients of a physically relevant quantity (see e.g. [28,29]) and is based on WENO schemes together with a sparse point representation (SPR). This version of the MR technique leads to an efficient and accurate scheme for multi-species kinematic flow models, which form the general class of models that includes our Model 2 (see [12]). The method employs interpolating wavelets [17, 26], which are efficiently combined with thresholding strategies to produce sparse approximations 
on a nearly optimal grid.

\subsection{Outline of the paper}

In Section 2 the MR scheme is described. In Section 2.1, we briefly recall the wellknown MR framework by Harten $[25,26]$ for the representation of a function by its point values on a sequence of nested dyadic grids. In doing so, we keep the same notation as in our previous work $[12,13,32]$, to which we refer for details. Several variants of MR methods applied to solving a differential equation differ in the evaluation of the differential operator. In this work, we consider the adaptive WENO scheme proposed in [12] to evolve the solution on a non-equidistant sparse grid via the concepts of local scale and of a sparse point representation (SPR) of a function, which go back to Holmström [28]. In Section 2.2 we recall the concept of an SPR of a function. The SPR of the solution is updated after each time step (of one application of an adaptive WENO scheme). The update of the SPR is briefly addressed in Section 2.3. The reference numerical scheme, namely a third-order WENO approximation built from flux vectors with Lax-Friedrichs splitting combined with a TVD Runge-Kutta scheme, is outlined in Section 2.4. The final MR algorithm is presented in Section 2.5.

In Section 3, the prototype hyperbolic-elliptic system from [22] is introduced (Section 3.1) and numerical simulations for Model 1 with two different cases of initial data (Examples 1 and 2) are presented (Section 3.2). In Section 4, an ellipticity analysis and numerical simulations for Model 2 are presented. Model 2 emerges from a general theory of sedimentation of polydisperse suspensions with $N$ particle species, which is presented in detail in $[6,9,11]$ and is briefly outlined in Section 4.1 for the special case $N=2$ we are interested in. This model for $N=2$ still involves three parameters, namely the exponent $n$ of the hindrance factor, the ratio $\gamma$ of the densities of both particles (relative to the fluid), and the squared ratio $\delta$ of the sizes of the smaller and the larger particles. The known properties of loss of hyperbolicity of the model for particles with different densities, that is, the hyperbolic-elliptic type behaviour for $N=2$, are reviewed in Section 4.2. The size, location and shape of the elliptic region depend on the values of these parameters. In particular, we provide exact expression of the points of tangency of the border of the elliptic region with that of the phase space. To prove that the region of ellipticity is indeed the interior of one closed curve, we consider in Section 4.4 the symmetric case of particles of equal size $(\delta=1)$ and a "heavy-buoyant" system with $\gamma=1$. It is also for this case that we present, in Section 4.5, MR simulations of oscillatory solutions. Examples 3, 4, 5, and 6 correspond to the respective values

$$
n=4, \quad n=8, \quad n=12, \quad \text { and } n=4.65,
$$

the latter of which corresponds to real experimental data [35]. In general, it turns out that permanently oscillatory solutions appear if initial data are chosen inside the elliptic region. Finally, some general conclusions are collected in Section 5; in particular we comment on the realism of oscillatory solutions for Model 2. 


\section{The multiresolution scheme}

\subsection{Multiresolution framework}

Let $\left(G^{0}, G^{1}, \ldots, G^{L_{c}}\right)$ denote a family of uniform nested grids on the interval $I:=[a, b]$, where $G^{0}:=\left(x_{0}^{0}, x_{1}^{0}, \ldots, x_{N_{0}}^{0}\right)$ with $N_{0}=2^{m}, m \in \mathbb{N}$ is the finest one corresponding to the finest resolution level, and $h_{0}:=(b-a) / N_{0}$ is the finest cell length. The values of a function $u$ on $G^{0}$ are the input data. The remaining grids are obtained recursively as follows: given a grid $G^{k-1}$, we obtain $G^{k}$ by removing the even-indexed grid points. Therefore

$$
x_{j}^{k}=x_{2 j}^{k-1}, \quad \text { for } 0 \leqslant j \leqslant N_{k}=2^{m-k}, k=1, \ldots, L_{c} .
$$

Thus, the representation of $u$ on any coarser grid $G^{k}, k=1, \ldots, L_{c}$, can be obtained directly from the finest level $k=0$ :

$$
u_{j}^{k}=u\left(x_{j}^{k}\right)=u\left(x_{2^{k} j}^{0}\right)=u_{2^{k} j^{\prime}}^{0}, \text { for } 0 \leqslant j \leqslant N_{k} .
$$

The function value at $x_{2 j-1}^{k-1}$ is obtained from the interpolation of the $r=2 s$ consecutive points $\left(u_{j-s}^{k}, \ldots, u_{j+s-1}^{k}\right)$ by an $(r-1)$-th degree polynomial. For $r=4$ we obtain the following interpolation formula for the value $\tilde{u}_{2 j-1}^{k-1}$ :

$$
\tilde{u}_{2 j-1}^{k-1}=\frac{1}{16} \cdot \begin{cases}5 u_{0}^{k}+15 u_{1}^{k}-5 u_{2}^{k}+u_{3}^{k}, & \text { for } j=1, \\ -u_{j-2}^{k}+9 u_{j-1}^{k}+9 u_{j}^{k}-u_{j+1}^{k}, & \text { for } j=2, \ldots, N_{k}-1, \\ u_{N_{k}-3}^{k}-5 u_{N_{k}-2}^{k}+15 u_{N_{k}-1}^{k}+5 u_{N_{k}}^{k} & \text { for } j=N_{k} .\end{cases}
$$

The interpolation errors, known as details, are

$$
d_{j}^{k}:=u_{2 j-1}^{k-1}-\tilde{u}_{2 j-1}^{k-1}, \quad \text { for } 1 \leqslant j \leqslant N_{k} .
$$

Thus, with the knowledge of $u^{k}:=\left(u_{0}^{k}, u_{1}^{k}, \ldots, u_{N_{k}}^{k}\right)$ and $d^{k}:=\left(d_{0}^{k}, d_{1}^{k}, \ldots, d_{N_{k}}^{k}\right)$, we can exactly recover the representation of $u$ on $G^{k-1}$. Therefore there is a bijective linear transform, the so-called interpolating wavelet transform, between both resolution levels, associating $u^{k-1}$ to $\left(d^{k}, u^{k}\right)$. Applying successively this procedure for $1 \leqslant k \leqslant L_{c}$, we can recover the values of $u$ on the finest level of resolution from its values on the coarsest level $L_{c}$ and the sequence of all details from levels $L_{c}$ to 1:

$$
u^{0} \leftrightarrow\left(d^{1}, u^{1}\right) \leftrightarrow\left(d^{1}, d^{2}, u^{2}\right) \leftrightarrow \cdots \leftrightarrow\left(d^{1}, d^{2}, \ldots, d^{L_{c}}, u^{L_{c}}\right)=: u_{\mathrm{M}},
$$

where $u_{\mathrm{M}}$ is the multiresolution representation [25] of $u^{0} \equiv u$. The details $d^{k}$ contain information on the local smoothness of $u$. They will be used to flag the non-smooth parts of the solution in the adaptive numerical method.

Multiresolution schemes achieve data compression and reduction of computational effort by considering only details $d_{j}^{k}$ that are larger in absolute value than a leveldependent comparison value $\varepsilon_{k}$. This means that one computes the quantities

$$
\hat{d}_{j}^{k}:=\operatorname{tr}_{\varepsilon_{k}}\left(d_{j}^{k}\right):=\left\{\begin{array}{ll}
0, & \text { if }\left|d_{j}^{k}\right|<\varepsilon_{k}, \\
d_{j}^{k}, & \text { if }\left|d_{j}^{k}\right| \geqslant \varepsilon_{k},
\end{array} \quad 1 \leqslant j \leqslant N_{k}, \quad 1 \leqslant k \leqslant L_{c},\right.
$$


where $\operatorname{tr}_{\varepsilon_{k}}$ is the so-called thresholding or truncation operator with the threshold parameters $\varepsilon_{k}, 1 \leqslant k \leqslant L_{c}$. We here utilize the thresholding strategy $\varepsilon_{k}=2^{-k} \varepsilon_{0}$, along with a given value $\varepsilon_{0}$, see [12].

In our case, the thresholding operation yields a set of markers (Boolean flags) that indicate whether $\left|d_{j}^{k}\right|>\varepsilon_{k}$ or not, that is, whether a position $(j, k)$ in the MR representation is significant or not. This information controls which point values of $u$ are included in the sparse point representation (SPR) of $u$. The computation of this set of markers is formalized in [25], where an algorithm for the scalar case is presented. In [12] Harten's algorithm is adapted for the vectorial case.

\subsection{Sparse point representation}

According to [28], the SPR of a function $u$ is associated to a sparse grid $\bar{\Gamma}$ such that $G^{L_{c}} \subset \bar{\Gamma} \subset G^{0}$, and consists of all exact point values of $u$ that correspond to positions belonging to $\bar{\Gamma}$. The set $\bar{\Gamma}$ consists of all positions judged significant according to (2.3), plus all point values of the coarsest grid, and certain safety points, which ensure that the SPR properly captures the finite speed of propagation of information and the formation of shock waves.

To build the SPR, we use the same sparse data structure as in [12], which is managed by the index set $\overline{\mathcal{D}}$ that flags whether a detail is significant or not. Therefore, $\bar{\Gamma}$ is the set of grid points flagged by $\overline{\mathcal{D}}$. The SPR of the vector-valued solution of (1.1) is based on the union of the SPRs of both vector components, since each solution component can be supported, and develop discontinuities, in different subregions of the domain. This behaviour permits a simplification similar to the one proposed in [14] for the Euler equations, where the SPR of the density is sufficient to capture the variations of the smoothness of all remaining vector components of the solution.

Consequently, to obtain the unified representation, we first compute index sets $\overline{\mathcal{D}}^{1}$ and $\overline{\mathcal{D}}^{2}$, one for each component, that include the positions of the significant coefficients and the positions of safety points, and then define $\overline{\mathcal{D}}:=\overline{\mathcal{D}}^{1} \cup \overline{\mathcal{D}}^{2}$. Then we build an SPR for each vector component with respect to $\bar{\Gamma}$, which is the set of grid points from $\overline{\mathcal{D}}$ plus all points from the coarsest level, i.e. we define

$$
\begin{aligned}
\bar{\Gamma} & :=\left\{x_{2 j-1}^{k-1} \mid(j, k) \in \overline{\mathcal{D}}\right\} \cup\left\{x_{0}^{L_{c}}, \ldots, x_{L_{c}}^{L_{c}}\right\} \\
& =\left\{x_{2 j-1}^{k-1} \mid(j, k) \in \overline{\mathcal{D}}^{1} \cup \overline{\mathcal{D}}^{2}\right\} \cup\left\{x_{0}^{L_{c}}, \ldots, x_{L_{c}}^{L_{c}}\right\} .
\end{aligned}
$$

(See [12] for further information and the corresponding algorithms.)

Whenever needed for flux evaluations, values corresponding to positions not contained in the SPR are interpolated from solution values belonging to the SPR. In other words, values of positions not belonging to the SPR can be discarded, so that the SPR presents a way of using the compression capabilities of MR representations. The compression effect due to the computation can be quantified by the number of details contained in the SPR, denoted $N_{\mathrm{s}}:=\# \bar{\Gamma}$. Of course, for a given function $u, N_{\mathrm{s}}$ depends substantially on the thresholding strategy. 


\subsection{Updating the SPR of the numerical solution}

The initial vector

$$
\Phi(x, 0)=\Phi_{0}(x):=\left(\phi_{1,0}(x), \phi_{2,0}(x)\right)^{\mathrm{T}}, \quad \Phi_{0}(x) \in \mathcal{E}_{\phi_{\max }}
$$

is discretized initially on the finest mesh $G^{0}$. For the first time step, the unified SPR will be obtained from the SPRs of each one of its components, i.e.

$$
u_{i}^{0}:=\left(\phi_{i, 0}\left(x_{0}^{0}\right), \phi_{i, 0}\left(x_{1}^{0}\right), \ldots, \phi_{i, 0}\left(x_{N_{0}}^{0}\right)\right), \quad i=1,2 .
$$

In the second and all subsequent time steps of the WENO-MRS method, we need to update the SPR from the respective previous time step. To obtain a new SPR after a time iteration is completed (here, a TVD Runge-Kutta cycle), we utilize an algorithm proposed in [28], which performs the wavelet transform only for those points that correspond to significant positions, i.e. positions in $\overline{\mathcal{D}}$. To compute the predictor, if any point value is needed that is not included in the SPR, the value is recursively interpolated from a coarser scale. The algorithm will terminate, since all function values of the coarsest grid belong to the SPR at any time step. After this sparse wavelet transform, the thresholding and extension operations are performed in order to update the flags. New point values to be included in the SPR (if necessary) are obtained in the same way by interpolation from coarser levels.

The update of the solution from $t_{n-1}$ to $t_{n}$ is performed for the solution values

$$
\phi_{i}^{n}\left(x_{j}^{k}\right) \approx \phi_{i}\left(x_{j}^{k}, t_{n}\right), \quad \text { for }(j, k) \in \overline{\mathcal{D}} \subset \bar{\Gamma}, \quad i=1,2,
$$

where $\bar{\Gamma}$ is the sparse grid of SPR at $t_{n-1}$. After each time step, the SPR update process is repeated for each solution component: new details are computed, new flags are determined, and according to the new flags, positions are included on or excluded from the updated sparse grid. In the case of inclusion, interpolated point values are associated to the new grid position. Once updated versions of both sets $\overline{\mathcal{D}}^{1}$ and $\overline{\mathcal{D}}^{2}$ are available, the new sparse grid $\bar{\Gamma}$ is defined by (2.4).

\subsection{Time and space discretizations}

We assume that the SPR of the numerical solution to be advanced over the next time step is already built, as explained in Section 2.3, where the sparse grid is denoted by $\bar{\Gamma}$. To simplify notation, we denote by $\bar{\Phi}=\left(\bar{\phi}_{1}, \bar{\phi}_{2}\right)^{\mathrm{T}}$ the SPR of the solution vectors.

For the time discretization of the sample system

$$
\partial_{t} \bar{\Phi}=\mathcal{L}(\bar{\Phi}) \equiv-\partial_{x} f(\bar{\Phi}),
$$

we utilize the TVD Runge-Kutta scheme [46] of order and step number $n_{\mathrm{RK}}=3$ :

$$
\begin{aligned}
& \bar{\Phi}_{j}^{(1)}=\bar{\Phi}_{j}^{n}+\Delta t \mathcal{L}_{j}\left(\bar{\Phi}^{n}\right), \quad \bar{\Phi}_{j}^{(2)}=\frac{1}{4}\left(3 \bar{\Phi}_{j}^{n}+\bar{\Phi}_{j}^{(1)}+\Delta t \mathcal{L}_{j}\left(\bar{\Phi}^{(1)}\right)\right), \\
& \bar{\Phi}_{j}^{n+1}=\frac{1}{3}\left(\bar{\Phi}_{j}^{n}+2 \bar{\Phi}_{j}^{(2)}+2 \Delta t \mathcal{L}_{j}\left(\bar{\Phi}^{(2)}\right)\right), j=0, \ldots, N_{0}, n=0,1,2, \ldots
\end{aligned}
$$


The numerical fluxes that determine the evolution of (1.1) will be calculated on $\bar{\Gamma}$. A conservative semi-discrete approximation

$$
\bar{\Phi}_{i}(t)=\left(\bar{\phi}_{1, i}(t), \bar{\phi}_{2, i}(t)\right)^{\mathrm{T}}
$$

to the exact solution $\bar{\Phi}\left(x_{i}, t\right)$ of (1.1) satisfies the system of ODEs

$$
\frac{d \bar{\Phi}_{i}}{d t}+\frac{1}{\overline{\Delta x}_{i}}\left(\widehat{f}_{i+1 / 2}-\widehat{f}_{i-1 / 2}\right)=0,
$$

where $\widehat{f}_{i+1 / 2}$ is the numerical flux associated with $x_{i}+\Delta x_{i+1} / 2$, where $x_{i} \in \bar{\Gamma}$ and $\overline{\Delta x}_{i}:=\left(\Delta x_{i+1}+\Delta x_{i}\right) / 2$. To guarantee flux upwinding, we apply a Lax-Friedrichs flux splitting to each component of the exact flux function:

$$
f(\Phi)=f^{+}(\Phi)+f^{-}(\Phi), \quad f^{ \pm}(\Phi):=\frac{1}{2}(f(\Phi) \pm \alpha \Phi) .
$$

The parameter $\alpha$ should equal the spectral radius of $\mathcal{J}_{f}(\Phi)$. For the case of Model 1, we use $\alpha=1$ as starting point, then after the first iteration set

$$
\alpha=\max _{i \in \bar{\Gamma}}\left(\max \left\{\left|\lambda_{1}\left(\bar{\Phi}_{i}\right)\right|,\left|\lambda_{2}\left(\bar{\Phi}_{i}\right)\right|\right\}\right),
$$

for values where $\lambda_{1}$ and $\lambda_{2}$ are the eigenvalues of the Jacobian of that model (see (3.4) in Section 3). For Model 2, the fluxes are of the "kinematic flow" type $f_{i}(\Phi)=\phi_{i} v_{i}(\Phi)$ for $i=1,2$, where $v_{i}$ is a bounded velocity function (see Section 4.1), we employ the following formula, which corresponds to the case $N=2$ of [12, Eq. (2.10)] for kinematic flow models with a general number $N$ of species:

$$
\alpha=\max _{i \in \bar{\Gamma}}\left(\max \left\{\left|v_{1}\left(\bar{\Phi}_{i}\right)\right|,\left|v_{2}\left(\bar{\Phi}_{i}\right)\right|\right\}\right) .
$$

Note that $\alpha$ is determined anew in each time step. Consequently, the value of $\Delta t$ is also corrected in each time step respecting the condition

$$
\alpha \Delta t / \overline{\Delta x}_{i} \leqslant \mathrm{CFL}
$$

where CFL is the CFL number.

The numerical flux is obtained as sum of the WENO approximations for each flux splitting components:

$$
\widehat{f}_{i+1 / 2}=f_{i+1 / 2}^{+, \text {WENO }}+f_{i+1 / 2}^{-, \text {WENO }} \text {. }
$$

The adaptive WENO scheme is described in detailed formulation in [12], and the original version for uniform grids, in [52].

As pointed out in [12], the numerical fluxes are computed from data of the same refinement level (local scale) such that all points involved in the computation of one WENO flux vector have the same distance

$$
d_{i}=\min \left\{\Delta x_{i}, \Delta x_{i+1}\right\}
$$


If any point is missing, we interpolate the corresponding solution value from a coarser scale and obtain the necessary flux components. (This procedure is well defined, since all values from the coarsest grid always belong to the SPR.) Values obtained by interpolation are only auxiliary values to keep the stencil locally uniform. The WENO approximations are computed exactly as is described in the literature, see e.g., [46, Ch. 2]; details are omitted here. Due to the conservativity of the method it is ensured that the total mass of each species is conserved.

\subsection{Multiresolution algorithm}

Now we present the MR scheme as an operation on the sparse grid $\bar{\Gamma}$, in which numerical fluxes are computed by the adaptive WENO procedure. We calculate the approximate solutions $\bar{\Phi}^{n, 0}, n=1,2, \ldots$, until the final time $T$ is reached, by Algorithm 2.3 of [12], which is recalled here.

\section{Algorithm 2.1}

$t \leftarrow 0$

while $t \leqslant T$ do

1. $\Phi^{n}(x)$ is given on the finest grid $(n=0)$ or on a sparse grid $(n>0)$. Calculate its SPR associated to $\bar{\Gamma}$ with $N_{\mathrm{s}}+1$ points.

2. Compute the local scale for each point of $\bar{\Gamma}$ and if necessary, the auxiliary neighbors to compute the numerical fluxes with locally uniform stencils.

3. Compute $\alpha$ (by (2.8) and (2.9) for Models 1 and 2, respectively) and $\Delta t \leftarrow \operatorname{CFL} \overline{\Delta x}_{i} / \alpha$, considering the smallest scale present on $\bar{\Gamma}$ and respecting the CFL condition. Set $t \leftarrow t+\Delta t$.

4. $\bar{\Phi}_{j}^{(0)} \leftarrow \Phi_{j}^{n, 0}, \quad x_{j} \in \bar{\Gamma}$.

do $i=1, \ldots, n_{\mathrm{RK}}$ (at this point, the values $\bar{\Phi}_{j}^{(0)}, \ldots, \bar{\Phi}_{j}^{(i-1)}$ for $x_{j} \in \bar{\Gamma}$ are known)

do $k=0, \ldots, i-1$

$$
\begin{aligned}
& \overline{\mathcal{L}}_{0}\left(\bar{\Phi}^{(k)}\right) \leftarrow-\frac{1}{\overline{\Delta x}_{0}} \widehat{f}_{1 / 2}^{0}, \quad \overline{\mathcal{L}}_{N_{\mathrm{s}}}\left(\bar{\Phi}^{(k)}\right) \leftarrow \frac{1}{\overline{\Delta x}_{N_{\mathrm{s}}}} \widehat{f}_{N_{\mathrm{s}}-1 / 2}^{0}, \\
& \overline{\mathcal{L}}_{j}\left(\bar{\Phi}^{(k)}\right) \leftarrow-\frac{1}{\overline{\Delta x}_{j}}\left(\widehat{f}_{j+1 / 2}^{0}-\widehat{f}_{j-1 / 2}^{0}\right), \quad x_{j} \in \bar{\Gamma} \backslash\left\{x_{0}, x_{N_{\mathrm{s}}}\right\},
\end{aligned}
$$

enddo

$$
\bar{\Phi}_{j}^{(i)} \leftarrow \sum_{k=0}^{i-1}\left(\alpha_{i k} \bar{\Phi}_{j}^{(k)}+\Delta t \beta_{i k} \overline{\mathcal{L}}_{j}\left(\bar{\Phi}^{(k)}\right)\right), \quad x_{j} \in \bar{\Gamma},
$$

enddo

$\bar{\Phi}_{j}^{n+1,0} \leftarrow \bar{\Phi}_{j}^{\left(n_{\mathrm{RK}}\right)}, \quad x_{j} \in \bar{\Gamma}$,

$n \leftarrow n+1$

endwhile

We herein consider initial-boundary value problems with zero-flux boundary conditions. In this case, the interpolating wavelet transform and the WENO scheme are 
modified at the boundaries in order to consider all information inside the domain. Nevertheless Algorithm 2.1 still holds under these modifications. Our interest is, however, focused on features of the solution in the interior of the computational domain, which are not affected (at least for the small times we are interested in) by the choice of boundary conditions.

\section{A prototype hyperbolic-elliptic system (Model 1)}

\subsection{Description of Model 1}

This model is a prototype of a mixed system of conservation laws modeling threephase flow in porous media [22] and is given by

$$
w_{t}+g(w)_{x}=0
$$

for the unknowns for $w=:(u, v)^{\mathrm{T}}$ with the flux function

$$
g(u, v):=\left(\frac{1}{\sqrt{3}}\left(\frac{v^{2}}{2}-\frac{u^{2}}{2}+v\right)+u, \frac{1}{\sqrt{3}}(u v-u)+v\right)^{\mathrm{T}} .
$$

The purpose of studying this system is that we wish to recover, by the MR method, the strong oscillations observed in [22] provided that the initial data $w(x, 0)=w_{0}(x)$, $x \in \mathbb{R}$ assume values from inside the elliptic region. In fact, we here assume that the initial data are chosen inside the region

$$
\nabla:=\left\{\boldsymbol{w}=(u, v)^{\mathrm{T}} \in \mathbb{R}^{2} \mid v<1, v \pm \sqrt{3} u \geq-2\right\}
$$

which has the property that $g(w) \in \nabla$ if $w \in \nabla$, which in turn implies that the solution $\boldsymbol{w}(\cdot, t)$ of the system will assume values in $\nabla$ whenever $\boldsymbol{w}_{0}(x) \in \nabla$ for all $x \in \mathbb{R}$. For this reason we call $\nabla$ an invariant region [22].

The eigenvalues of the Jacobian $\mathcal{J}_{g}$ are given by

$$
\lambda_{1,2}=1 \mp \frac{1}{\sqrt{3}} \sqrt{u^{2}+v^{2}-1},
$$

such that the system (3.1), (3.2) is of mixed type, being hyperbolic for

$$
\|\boldsymbol{w}\|_{2}^{2}=u^{2}+v^{2}>1,
$$

and elliptic for

$$
\boldsymbol{w} \in \mathcal{E}_{1}:=\left\{\boldsymbol{w}=(u, v)^{\mathrm{T}} \in \nabla \mid u^{2}+v^{2}<1\right\} .
$$

Note that $\mathcal{E}_{1}$ is tangent to each of the three sides of $\nabla$. Moreover, the absolute values of the eigenvalues are $\left|\lambda_{1}\right|=\left|\lambda_{2}\right|=1$ on the boundary of the elliptic region and increase toward the origin, where $\left|\lambda_{1}\right|=\left|\lambda_{2}\right|=2 \sqrt{3} / 3$. 


\subsection{Simulation of Model 1}

We now revisit the simulations made in [22] and try to find out where the oscillations come from. In [22] the initial datum chosen as a constant which is perturbed at a small number of grid points in the vicinity of $x=0$. In one case considered in [22] $\left(w_{0}=\left(u_{0}, v_{0}\right)^{\mathrm{T}}=(0.1,0.2)^{\mathrm{T}}\right.$ with the exception of a vicinity of $\left.x=0\right)$, oscillations can be observed, whereas in the other case of [22] $\left(w_{0}=\left(u_{0}, v_{0}\right)^{\mathrm{T}}=(-0.9,0.1)^{\mathrm{T}}\right.$ except for a vicinity of $x=0$ ) oscillations are not observed; rather, the solution consists of a small number of travelling discontinuities satisfying an extended Lax shock admissibility criterion (i.e., the usual Lax shock inequalities hold for the real parts of the eigenvalues).
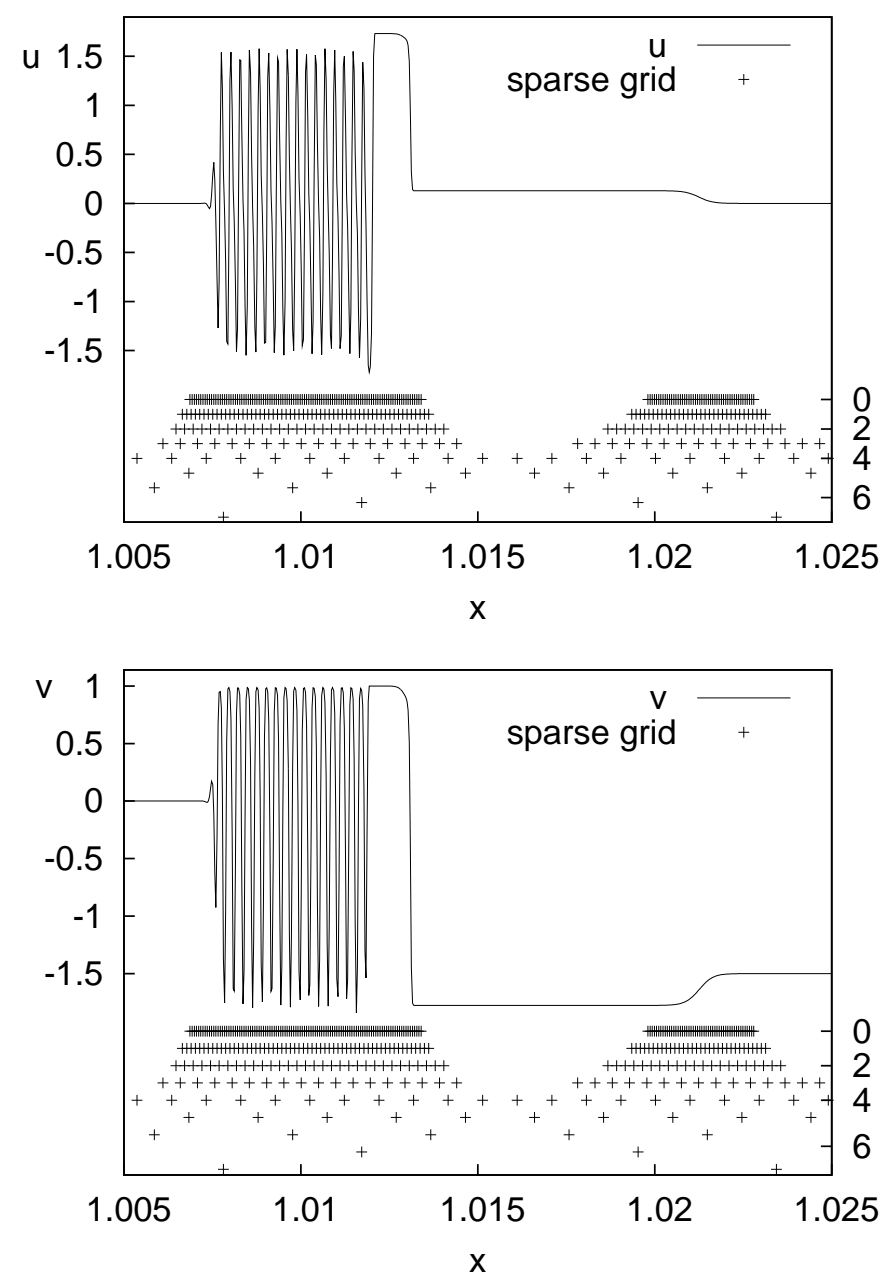

Figure 1: Example 1 (Model 1, Riemann problem): profiles of $u$ (top) and $v$ (bottom) at $t=0.0153$, with marked significant positions of the SPR. The CFL number is 0.125 , as in [22]. 


\subsubsection{Example 1: Model 1 with Riemann initial data}

To examine the anatomy of oscillations, we consider a Riemann problem for (3.1), (3.2) with the initial datum

$$
\boldsymbol{w}(x, 0)=\boldsymbol{w}_{0}(x):= \begin{cases}\boldsymbol{w}_{\mathrm{L}}=\left(u_{\mathrm{L}}, v_{\mathrm{L}}\right)^{\mathrm{T}}=(0,0)^{\mathrm{T}} \in \mathcal{E}_{1}, & \text { for } x<1, \\ \boldsymbol{w}_{\mathrm{R}}=\left(u_{\mathrm{R}}, v_{\mathrm{R}}\right)^{\mathrm{T}}=(0,-1.5)^{\mathrm{T}} \in \nabla \backslash \mathcal{E}_{1}, & \text { for } x \geqslant 1 .\end{cases}
$$

To focus on the development of oscillations the solution has been evolved only over a small number of time steps up to finite time $t=T=0.0153$. The corresponding profiles

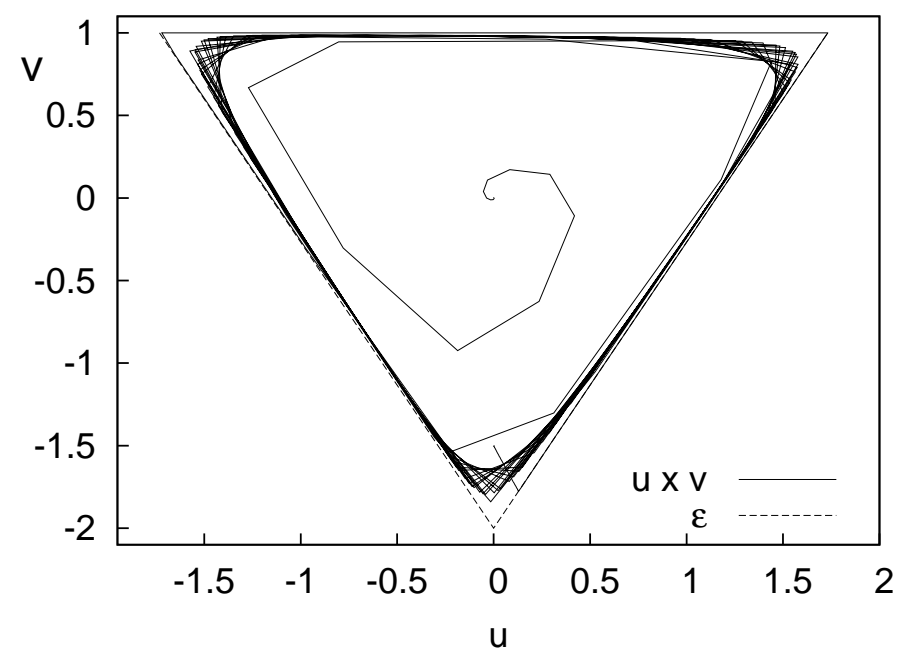

Figure 2: Example 1 (Model 1, Riemann problem): solution at $t=0.0153$ in the $(u, v)$-plane.

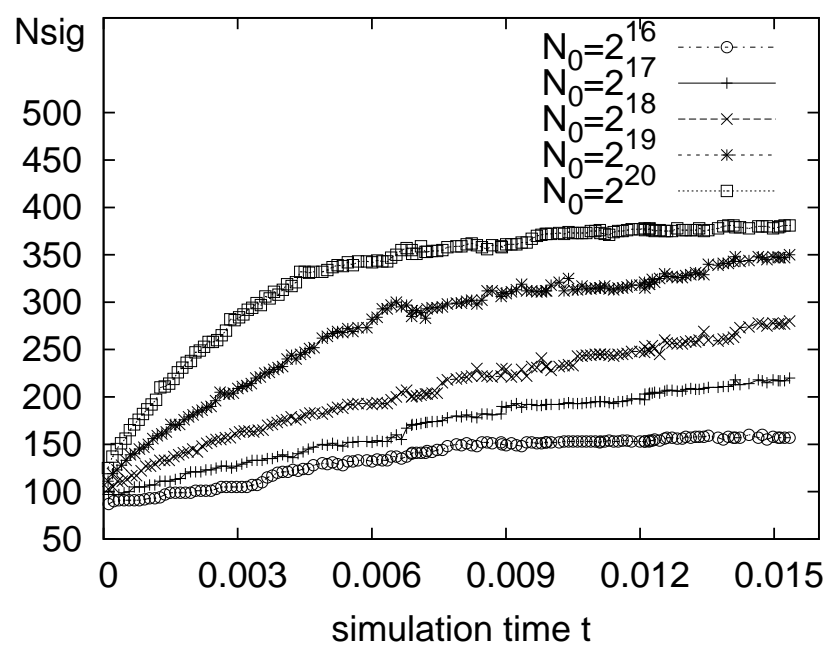

Figure 3: Example 1 (Model 1, Riemann problem): number of significant points, considering the finest resolution level with $N_{0}=2^{p}, p=16, \ldots, 20$ points. 
of the components $u$ and $v$ of the solution are shown in Fig. 1. Outside the shown range of positions $x$, the solution is constant. Both profiles present a mixed, partially oscillatory pattern composed by a smooth part of the solution followed by strong oscillations. In addition, Fig. 1 displays the momentary significant positions, that is, the levels of multiresolution occupied by the SPR of the solution, which are marked by '+++'; similar information is included in some of the plots of Figs. 9, 11, 12, 14, 15, 16 and 17 for Examples 2-6.

Fig. 2 shows the same solution as a trajectory in the $(u, v)$-plane connecting the points $\left(u_{\mathrm{L}}, v_{\mathrm{L}}\right)$ and $\left(u_{\mathrm{R}}, v_{\mathrm{R}}\right)$. Apparently, the solution seems to avoid the elliptic region $\mathcal{E}_{1}$. In preliminary computations (not shown here) it turned out that when the Riemann initial data of the previous examples are interchanged (i.e., $w_{\mathrm{L}}=(0,-1.5)^{\mathrm{T}}$, $\left.w_{R}=(0,0)^{\mathrm{T}}\right)$, then the spirals have the same clockwise orientation and the propagation speed is also positive.
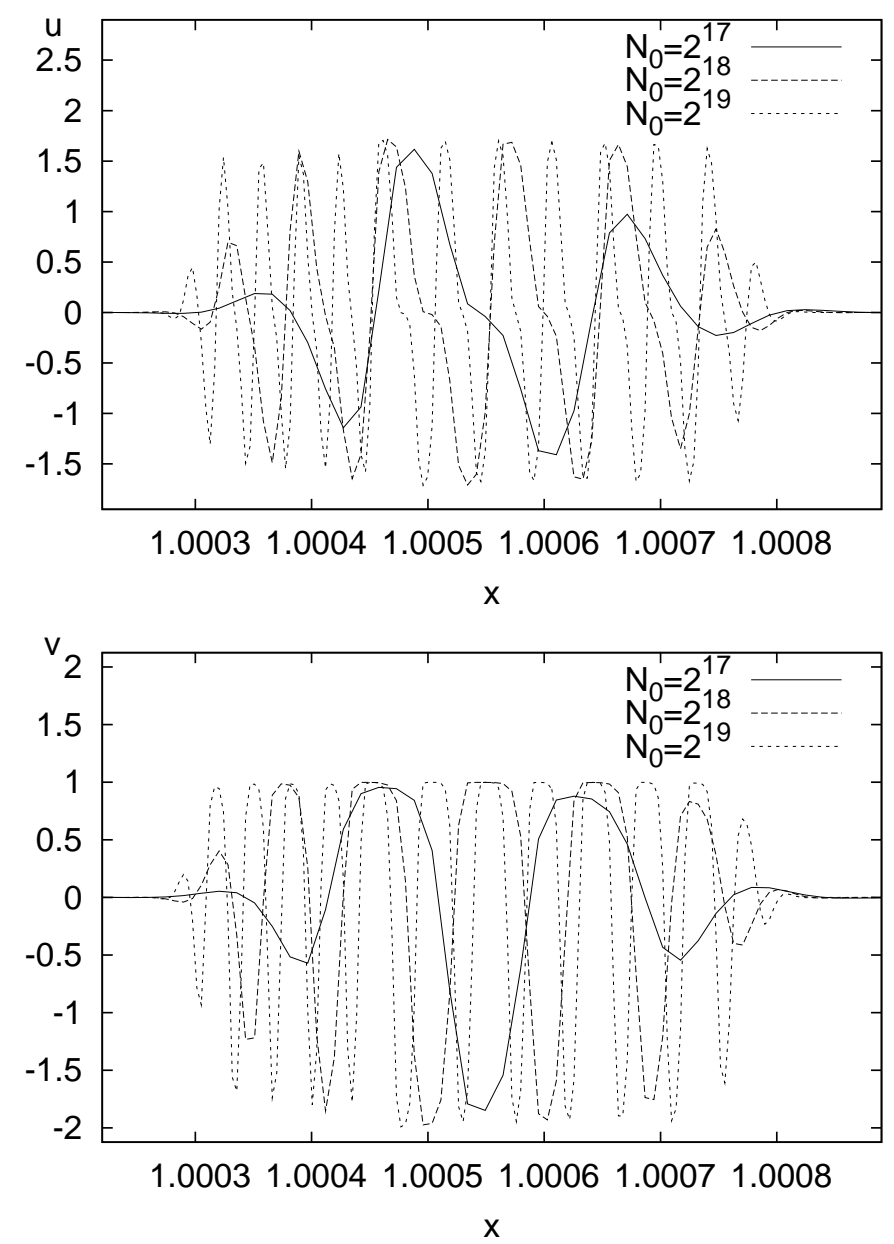

Figure 4: Example 2 (Model 1, almost constant initial data): profile of oscillations of $u$ (top) and $v$ (bottom) with refinements considering the finest resolution level of the multiresolution with $2^{p}$ points, $p=17,18,19$. 
Finally, Fig. 3 illustrates the number of significant points used by the MR scheme for fine levels from $2^{16}$ to $2^{20}$. The number of significant points maintained by the MR representation is kept stable and at the same magnitude for all resolutions. This is an important feature of the adaptive scheme, indicating that significant positions are concentrated exactly where the solution varies strongly. The threshold value $\varepsilon_{0}$ considered for each number of point in the finest grid $N_{0}=2^{p}, p=16, \ldots, 20$, was $\varepsilon_{0}=5 \times 10^{12-p}$. Note that these numbers correspond to the subdivision of the interval $[a, b]=[0,2]$ into $N_{0}$ subintervals.

\subsubsection{Example 2: Model 1 with almost constant initial data}

In Example 2, we simulate again Model 1, but choose the initial datum $w_{0}(x)=(0,0)^{\mathrm{T}}$ with the exception of a few grid points near the origin; we refer to this as "almost constant" initial data. Fig. 4 compares numerically computed solution profiles corre-
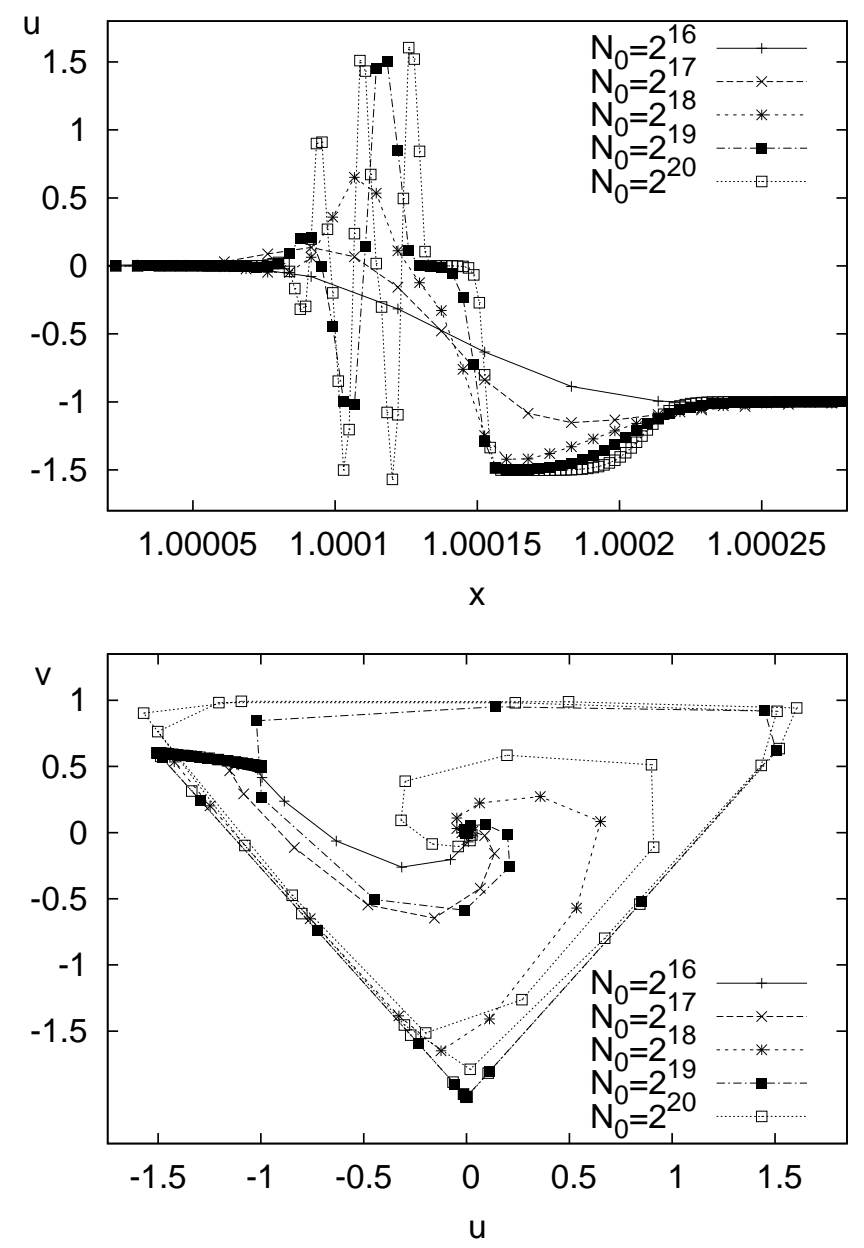

Figure 5: Example 2 (Model 1, almost constant initial data): solution after a few time steps for fine multiresolution scales varying from $2^{16}$ to $2^{20}$. Top: profile of $u$. Bottom: solution in phase space. 
sponding to various numbers of points provided in the finest MR level. We observe that the oscillation frequency of the numerical solution depends on the meshsize of the finest grid. Specifically, we roughly observe that if the number of discrete cells is doubled, then the number of appearing peaks is also doubled. Nevertheless, the oscillations maintain the same patterns for different meshwidths of the finest mesh. Fig. 5 presents the spiraling dynamics after a few time steps both by a profile of $v$ and in the phase space $u, v$. Increasing the resolution does not lead to some kind of convergence to a classical solution, but only increases the strength of oscillations. Here and in Examples 3-6 for Model 2, the number $N_{0}$ corresponds to the subdivision of the interval $[a, b]=[0,1]$ into $N_{0}$ subintervals.

\section{Settling of heavy-buoyant bidisperse suspensions (Model 2)}

\subsection{Description of Model 2}

The goal of examining Model 2 is to analyze whether oscillations similar to these observed for Model 1 also appear in another example of multiphase flow. The comparison gives physical insights on the conditions for oscillatory solution behaviour.

We consider a suspension composed of rigid spherical particles which are dispersed in a viscous fluid of density $\varrho_{\mathrm{f}}$ and viscosity $\mu_{\mathrm{f}}$. In the general case, the particles belong to $N$ different species having sizes (diameters) $d_{1} \geqslant d_{2} \geqslant \ldots \geqslant d_{N}$ and densities $\varrho_{1}, \ldots, \varrho_{N}$, where $d_{i} \neq d_{j}$ or varrho ${ }_{i} \neq \varrho_{j}$ for $i \neq j$. Model equations for the threedimensional motion of the mixture were derived in [11] from the mass and linear momentum balances for the fluid and each solid species by introducing constitutive assumptions and simplifying the model equations as a consequence of a dimensional analysis. The one-dimensional version of the model goes back to Masliyah [42] and Lockett and Bassoon [38], and is therefore frequently referred to as "MLB model". The relevant parameters are $\delta_{i}:=d_{i}^{2} / d_{1}^{2}$ and $\bar{\varrho}_{i}:=\varrho_{i}-\varrho_{\mathrm{f}}$ for $i=1, \ldots, N$. Moreover, we introduce the vector $\bar{\varrho}:=\left(\bar{\varrho}_{1}, \ldots, \bar{\varrho}_{N}\right)^{\mathrm{T}}$, the total solids fraction $\phi:=\phi_{1}+\cdots+\phi_{N}$, the

viscosity parameter $\mu:=-g d_{1}^{2} /\left(18 \mu_{\mathrm{f}}\right)<0$, where $g$ is the acceleration of gravity, and the hindered settling factor $V=V(\phi)$, which may be chosen as

$$
V(\phi)= \begin{cases}(1-\phi)^{n-2}, & \text { if } 0 \leqslant \phi \leqslant \phi_{\max }, \quad n>2, \\ 0, & \text { otherwise },\end{cases}
$$

where $\phi_{\max }$ denotes a maximum solids volume fraction, which we here assume to be a constant. Then the phase velocity of particle species $i$ is

$$
v_{i}(\Phi)=\mu V(\phi)\left[\delta_{i}\left(\bar{\varrho}_{i}-\bar{\varrho}^{\mathrm{T}} \Phi\right)-\sum_{m=1}^{N} \delta_{m} \phi_{m}\left(\bar{\varrho}_{m}-\bar{\varrho}^{\mathrm{T}} \Phi\right)\right], \quad i=1, \ldots, N
$$

The present treatment is limited to the case $N=2$. Taking into account that $\delta_{1}=1$, we 
then obtain from (4.2) the equations

$$
\begin{aligned}
& v_{1}(\Phi)=\mu V(\phi)\left(\left(1-\phi_{1}\right)\left(\bar{\varrho}_{1}-\bar{\varrho}^{\mathrm{T}} \Phi\right)-\delta_{2} \phi_{2}\left(\bar{\varrho}_{2}-\bar{\varrho}^{\mathrm{T}} \Phi\right)\right), \\
& v_{2}(\Phi)=\mu V(\phi)\left(\left(1-\phi_{2}\right) \delta_{2}\left(\bar{\varrho}_{2}-\bar{\varrho}^{\mathrm{T}} \Phi\right)-\phi_{1}\left(\bar{\varrho}_{1}-\bar{\varrho}^{\mathrm{T}} \Phi\right)\right) .
\end{aligned}
$$

The relevant phase space for $N=2$ is

$$
\mathcal{D}_{\phi_{\max }}:=\left\{\Phi \in \mathbb{R}^{2} \mid \phi_{1} \geqslant 0, \quad \phi_{2} \geqslant 0, \quad \phi_{1}+\phi_{2} \leqslant \phi_{\max }\right\} .
$$

For one-dimensional batch settling of a suspension with initially given composition in a closed vessel of depth $L$, the governing equation is

$$
\begin{aligned}
& \partial_{t} \Phi+\partial_{x} f(\Phi)=0, \quad x \in(0, L), \quad t>0, \\
& f(\Phi)=\left(f_{1}(\Phi), f_{2}(\Phi)\right)^{\mathrm{T}}, \quad f_{1}(\Phi)=\phi_{1} v_{1}(\Phi), \quad f_{2}(\Phi)=\phi_{2} v_{2}(\Phi),
\end{aligned}
$$

where we consider the initial and zero-flux boundary conditions

$$
\begin{aligned}
& \Phi(x, 0)=\Phi_{0}(x), \quad x \in[0, L], \\
& \left.f\right|_{x=0}=\left.f\right|_{x=L}=0 .
\end{aligned}
$$

\subsection{Mixed type property}

For the model exposed in the previous section and $N=2$, the criterion for ellipticity is equivalent to the instability criterion by Batchelor and Janse van Rensburg [2]. In [11], it is shown that loss of hyperbolicity, that is the occurrence of complex eigenvalues of $\mathcal{J}_{f}(\Phi)$, provides an instability criterion for polydisperse suspensions for arbitrary $N$. Instability regions for $N=2,3$ and different choices of $f(\Phi)$ are determined in $[7,11]$. In [6], it is proved that for equal-density particles $\left(\bar{\varrho}_{1}=\ldots=\bar{\varrho}_{N}=\varrho_{\mathrm{s}}-\varrho_{\mathrm{f}}\right)$ and arbitrary particle size distributions with $\delta_{i} \neq \delta_{j}$ for $i \neq j$, the system (4.4) with the flux vector $f(\Phi)$ defined via (4.3) is strictly hyperbolic for all $\Phi \in \mathcal{D}_{1}$ with $\phi_{1}>0, \ldots, \phi_{N}>0$ and $\phi<1$. The instability criterion for one-dimensional batch settling is the same as for the full two- or three-dimensional model, in which the corresponding first-order system of conservation laws is coupled with additional equations of motion for the mixture.

The consequences of lack of stability include the formation of blobs and "fingers" in bidisperse sedimentation, increased sedimentation rates, decreased separation quality of hydraulic classifiers, and non-homogeneous sediments in material manufacturing by suspension processing [7]. These phenomena have indeed been observed in experiments (e.g., by Weiland et al. [50]) under the circumstances predicted by the instability criterion. On the other hand, the hyperbolicity, and thus stability result for equal-density spheres agrees with experimental evidence, since instabilities never have been observed with this type of mixtures, but always involve particles of different densities [50]. For one-dimensional kinematic models, such as ours, lack of stability may lead to anomalous behaviour of the numerical solution, for example to 


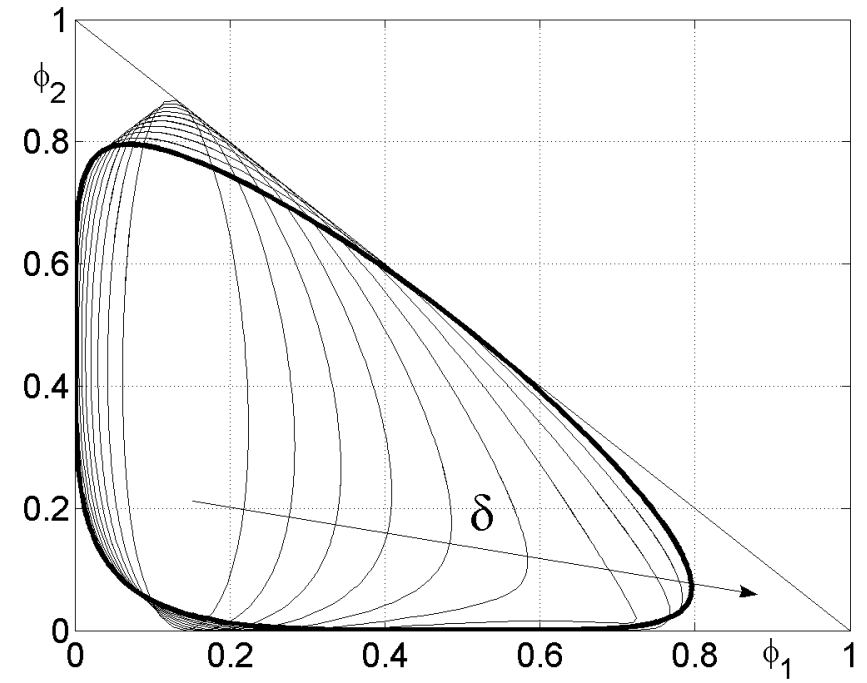

Figure 6: Shape of elliptic region $\mathcal{E}_{2}(n, \delta, \gamma)$ : reference region $\mathcal{E}_{2}(8,1,-1)$ and variation of the parameter $\delta \in\{0.1,0.2, \ldots, 0.8,0.9,1\}$. The arrow indicates how the closed curves are ordered with respect to increasing values of $\delta$.

oscillations or a "locking" effect, i.e., heavy and buoyant particles block each other within the vessel; such an example is presented in [5, Fig. 10]. (The issue of physical realism will be further addressed in Section 5.)

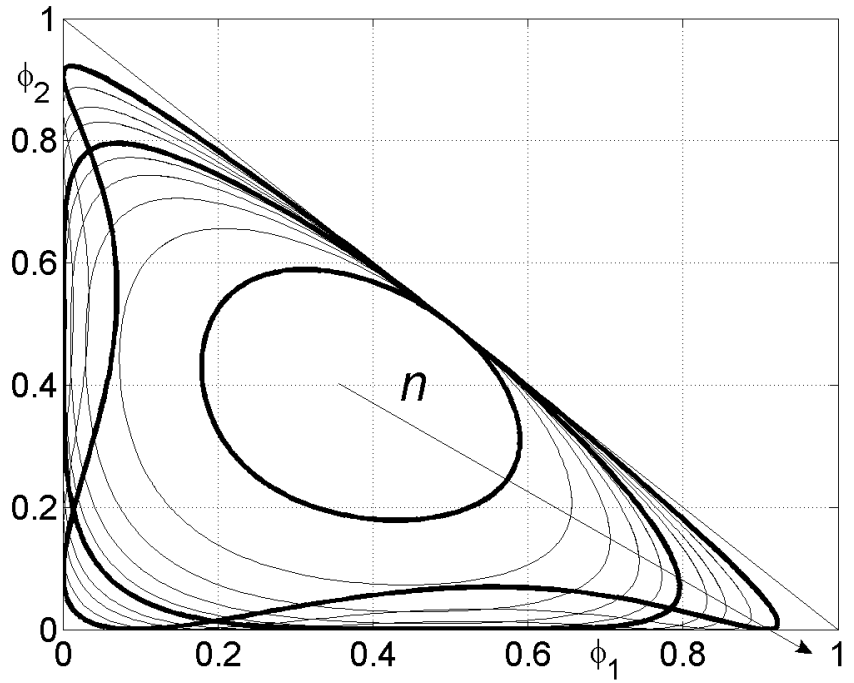

Figure 7: Shape of elliptic region $\mathcal{E}_{2}(n, \delta, \gamma)$ : reference region $\mathcal{E}_{2}(8,1,-1)$ and variation of the parameter $n \in\{3,4,5,6,7,8,10,12,16,24\}$. The arrow indicates how the closed curves are ordered with respect to increasing values of $n$. 
Finally, for our case $N=2$, it is convenient to introduce the parameter $\gamma:=\bar{\varrho}_{2} / \bar{\varrho}_{1}$, and we set $\delta:=\delta_{2}$. Introducing the scaled variable $\tilde{x}:=x /\left(\bar{\varrho}_{1} \mu\right)$ and immediately switching back to $x$ instead of $\tilde{x}$, we can rewrite (4.3), (4.4) in the form (1.1), where the fluxes are defined by

$$
\begin{aligned}
& f_{1}\left(\phi_{1}, \phi_{2}\right)=\phi_{1} V\left(\phi_{1}+\phi_{2}\right)\left(\left(1-\phi_{1}\right)\left(1-\phi_{1}-\gamma \phi_{2}\right)-\delta \phi_{2}\left(\left(1-\phi_{2}\right) \gamma-\phi_{1}\right)\right), \\
& f_{2}\left(\phi_{1}, \phi_{2}\right)=\phi_{2} V\left(\phi_{1}+\phi_{2}\right)\left(\delta\left(1-\phi_{2}\right)\left(\left(1-\phi_{2}\right) \gamma-\phi_{1}\right)-\phi_{1}\left(1-\phi_{1}-\gamma \phi_{2}\right)\right) .
\end{aligned}
$$

The results of $[6,11]$ (see also [10]) can then be stated as follows: for equal-density spheres, i.e., $\gamma=1$, arbitrary $\delta \in(0,1]$, and a hindered settling factor $V=V(\phi)$ with $V(\phi) \geqslant 0$ and $V^{\prime}(\phi)<0$ on $\left[0, \phi_{\max }\right)$ (for example, (4.1)) the system (1.1), (4.7) is always strictly hyperbolic in the interior of $\mathcal{D}_{\phi_{\max }}$. On the other hand, for $\gamma \neq 1$, the system (1.1), (4.7) may exhibit an appreciable ellipticity (instability) region $\mathcal{E}_{2}$.

In the remainder of the paper, we we limit the discussion to the hindered settling factor $V(\phi)$ given by (4.1). Consequently, the parameters that appear in our analysis are the exponent $n$, the reduced density ratio $\gamma$ and the squared size ratio $\delta$. We highlight the dependence of $\mathcal{E}_{2}$ on these parameters by writing $\mathcal{E}_{2}=\mathcal{E}_{2}(n, \gamma, \delta)$.

Values $n$ between 4 and 6 are supported by theoretical predictions and have been found suitable for many experimental systems; several works (e.g., [35]) utilize $n=4.65$. In [11] a selection of plots of $\mathcal{E}_{2}(4.65, \gamma, \delta)$ for various choices of $\gamma$ and $\delta$ are presented. It turns out that the ellipticity region is particularly large if $\gamma<0$, i.e., one of the species is "heavy", with a density larger than that of the fluid, and the other is "buoyant", with a density smaller than that of the fluid. It is the "heavy-buoyant" situation that is chosen for closer inspection in Examples 3, 4 and 5, while in Example 6 we set $\gamma=0$, corresponding to a suspension with heavy particles and a second "neutrally buoyant" species.

\subsection{Elliptic region of Model 2}

Numerical simulations indicate that the emergence of strong oscillations associated to the elliptic degeneracy is related to the location and shape of the elliptic region with respect to the invariance region. Strong oscillations only appear when $\mathcal{E}_{2}$ is tangent to each side of $\mathcal{D}_{\phi_{\max }}$.

For homogenous initial data $\Phi_{0}$ which are slightly perturbed in $\mathcal{E}_{2}$ there appears a spiral starting at $\Phi_{0}$ in the phase space. This spiraling indicates that perturbed data want to escape from the elliptic region. Such an unbounded growth can be proved for linearized systems: a Fourier transform in space shows that one component of the solution growths exponentially, leading to an unstable system [3]. Such a growth can only be limited by the size of the elliptic region.

The shape, location and points of tangency of $\mathcal{E}_{2}$ with respect to $\mathcal{D}_{\phi_{\max }}$ (the set of all possible solution values) decide whether an "escape" or "fadeout" of the oscillatory behavior is possible. In Model 1, the elliptic region $\mathcal{E}_{1}$ is tangent to each side of the 

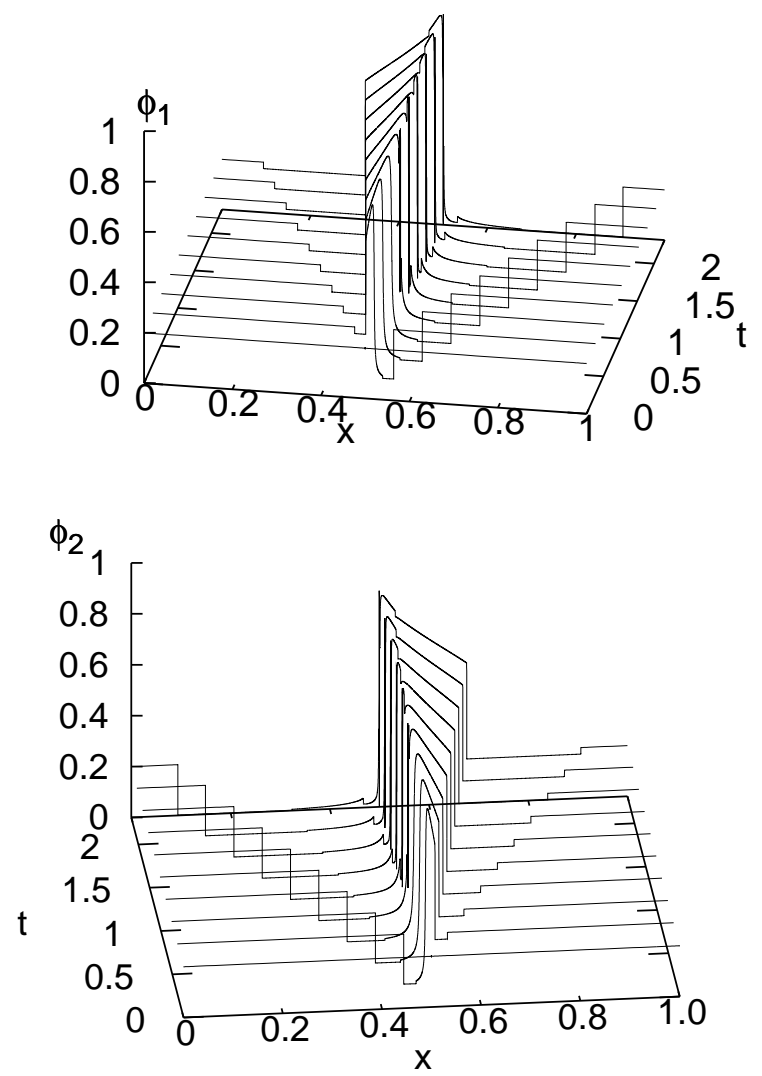

Figure 8: Example 3 (Model 2 with $\delta=1, \gamma=-1, n=4$ ): profiles of $\phi_{1}$ (top) and $\phi_{2}$ (bottom) at several times.

invariance region $\nabla$. The fact that the perturbed data inside $\mathcal{E}_{1}$ want to escape but can not leads to the wild oscillations.

To control (i.e., recover or avoid) the oscillatory behavior it is necessary to analyze the shape of $\mathcal{E}_{2}(n, \gamma, \delta)$ and in particular to develop a criterion when it is tangent to the boundary of $\mathcal{D}_{\phi_{\max }}$. To this end, the discriminant $\Delta=\Delta\left(\phi_{1}, \phi_{2}\right)$ defined by (1.3), where $f_{1}$ and $f_{2}$ are given by (4.7), is evaluated on the axes $\phi_{2}=0$ and $\phi_{1}=0$ as well as on the antidiagonal $\phi_{1}+\phi_{2}=1$. Noting that $J_{12} J_{21}=0$ if $\phi_{1}=0$ or $\phi_{2}=0$, we obtain the discriminants

$$
\begin{aligned}
& \Delta\left(\phi_{1}, 0\right)=\left(1-\phi_{1}\right)^{2 n-4}\left(n \phi_{1}^{2}+(\delta-1-n) \phi_{1}+1-\delta \gamma\right)^{2} \geqslant 0 \\
& \Delta\left(0, \phi_{2}\right)=\left(1-\phi_{2}\right)^{2 n-4}\left(\delta \gamma n \phi_{2}^{2}+(\gamma-\delta \gamma n-\delta \gamma) \phi_{2}+\delta \gamma-1\right)^{2} \geqslant 0 .
\end{aligned}
$$

We are interested in choosing the parameters $n, \gamma$ and $\delta$ in such a way that the boundary $\partial \mathcal{E}_{2}(n, \gamma, \delta)$ of $\mathcal{E}_{2}(n, \gamma, \delta)$ is tangent to the coordinate axes $\phi_{1}=0$ and $\phi_{2}=0$ (and to 

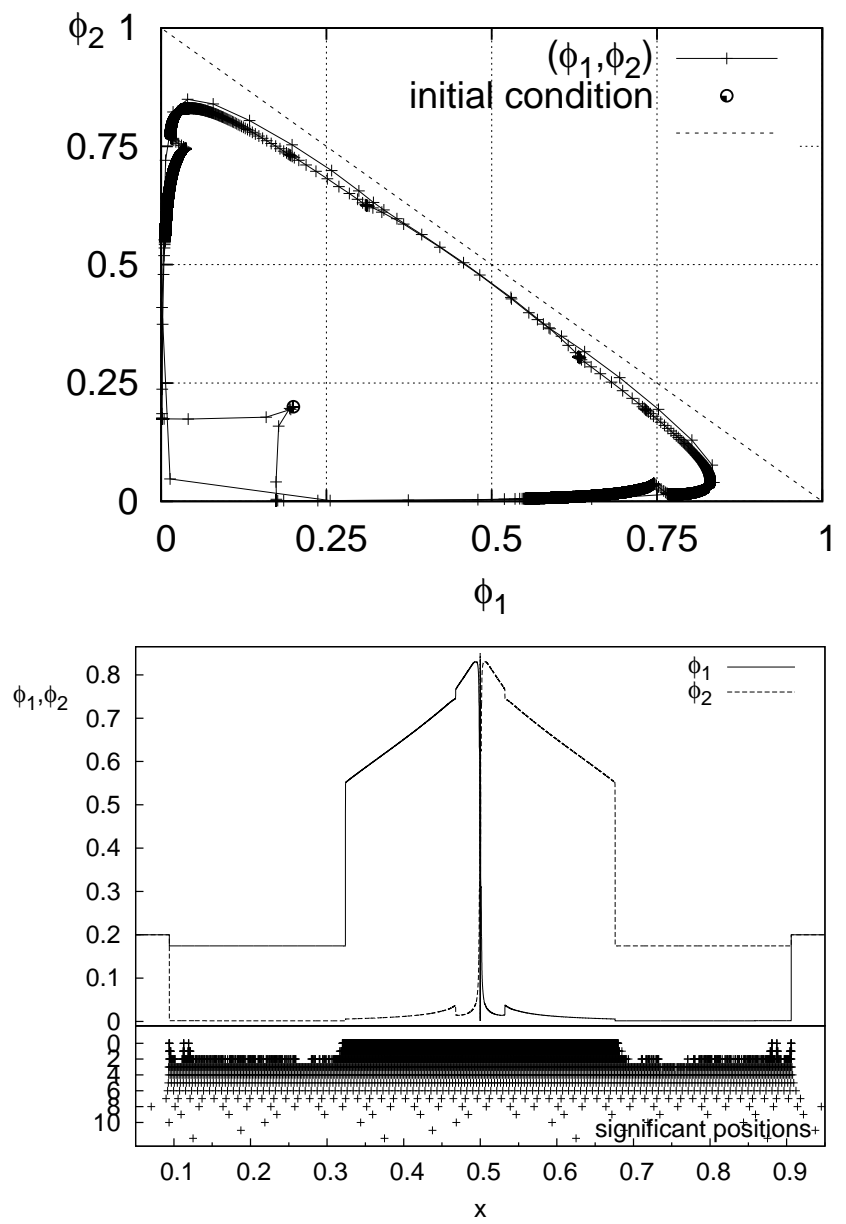

Figure 9: Example 3 (Model 2 with $\delta=1, \gamma=-1, n=4$ ). Top: phase space. Bottom: profiles of $\phi_{1}$ and $\phi_{2}$ and significant positions at $t=2.06$.

the anti-diagonal $\left.\phi_{1}+\phi_{2}=1\right)$. We seek solutions $0<\phi_{1}<1$ and $0<\phi_{2}<1$ of $\Delta\left(\phi_{1}, 0\right)=0$ and $\Delta\left(0, \phi_{2}\right)=0$, respectively. Using (4.8) and (4.9), we obtain the following candidate values for tangent points of $\partial \mathcal{E}_{2}(n, \gamma, \delta)$ with the coordinate axes:

$$
\begin{aligned}
& \phi_{1}=\frac{1}{2}+\frac{1-\delta}{2 n} \pm \frac{\sqrt{r_{1}(n, \gamma, \delta)}}{2 n}, \\
& r_{1}(n, \gamma, \delta):=(n-(1-\delta))^{2}+4 n \delta \gamma \\
& \phi_{2}=\frac{1}{2}+\frac{\delta-1}{2 \delta n} \pm \frac{\sqrt{r_{2}(n, \gamma, \delta)}}{2 \delta \gamma n}, \\
& r_{2}(n, \gamma, \delta):=\delta^{2} \gamma^{2} n^{2}+2 n\left(2 \delta \gamma-\delta \gamma^{2}-\delta^{2} \gamma^{2}\right)+\gamma^{2}(1-\delta)^{2} .
\end{aligned}
$$

Let us admit for a moment negative values of $\phi_{1}$ or $\phi_{2}$ for sake of the argument. Eqs. (4.10) and (4.11) tell us that if $r_{i}(n, \gamma, \delta)>0$, then there are two tangents or crossing 

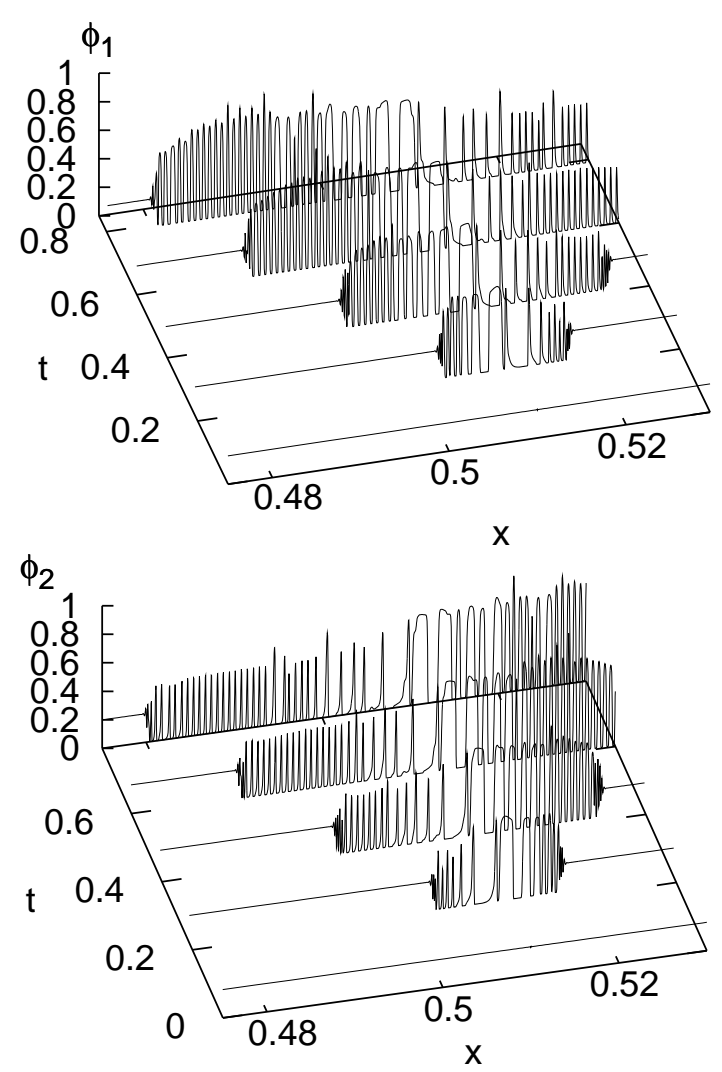

Figure 10: Example 4 (Model 2 with $\delta=1, \gamma=-1$ and $n=8$ ): profiles of $\phi_{1}$ (top) and $\phi_{2}$ (bottom) at several times.

points $\partial \mathcal{E}_{2}(n, \gamma, \delta)$ with the $\phi_{i}$-axis, $i=1,2$. However, it is impossible that $\partial \mathcal{E}_{2}(n, \gamma, \delta)$ crosses one of these axes, since this would imply that $\Delta<0$ on a segment of positive length on one of these axes, in contradiction to (4.8) and (4.9). We conclude that real solutions of (4.10) and (4.11) are tangent to the $\phi_{1}$-axis or the $\phi_{2}$-axis. Moreover, for $\gamma=0$ the axis $\phi_{1}=0$ never exhibits a tangent (since the left-hand side of (4.9) is then strictly positive for $0<\phi_{2}<1$ ), whereas on the axis $\phi_{2}=0$ always tangents can be enforced for sufficiently large $n$.

On the antidiagonal $\phi_{1}+\phi_{2}=1, \Delta$ always vanishes, such that a single root cannot be extracted. To get a hint on the structure of $\partial \mathcal{E}(n, \gamma, \delta)$ close to the antidiagonal, we write $\Delta\left(\phi_{1}, \phi_{2}\right)$ as

$$
\Delta\left(\phi_{1}, \phi_{2}\right)=\left(1-\phi_{1}-\phi_{2}\right) \tilde{\Delta}\left(\phi_{1}, \phi_{2}\right)
$$

and note that

$$
\tilde{\Delta}\left(1-\phi_{2}, \phi_{2}\right)=(\gamma-1)^{2}\left(\phi_{2}^{2}(n \delta-n)+\phi_{2}\left(n-n \delta^{2}-\delta-1\right)+\delta\right)^{2} .
$$



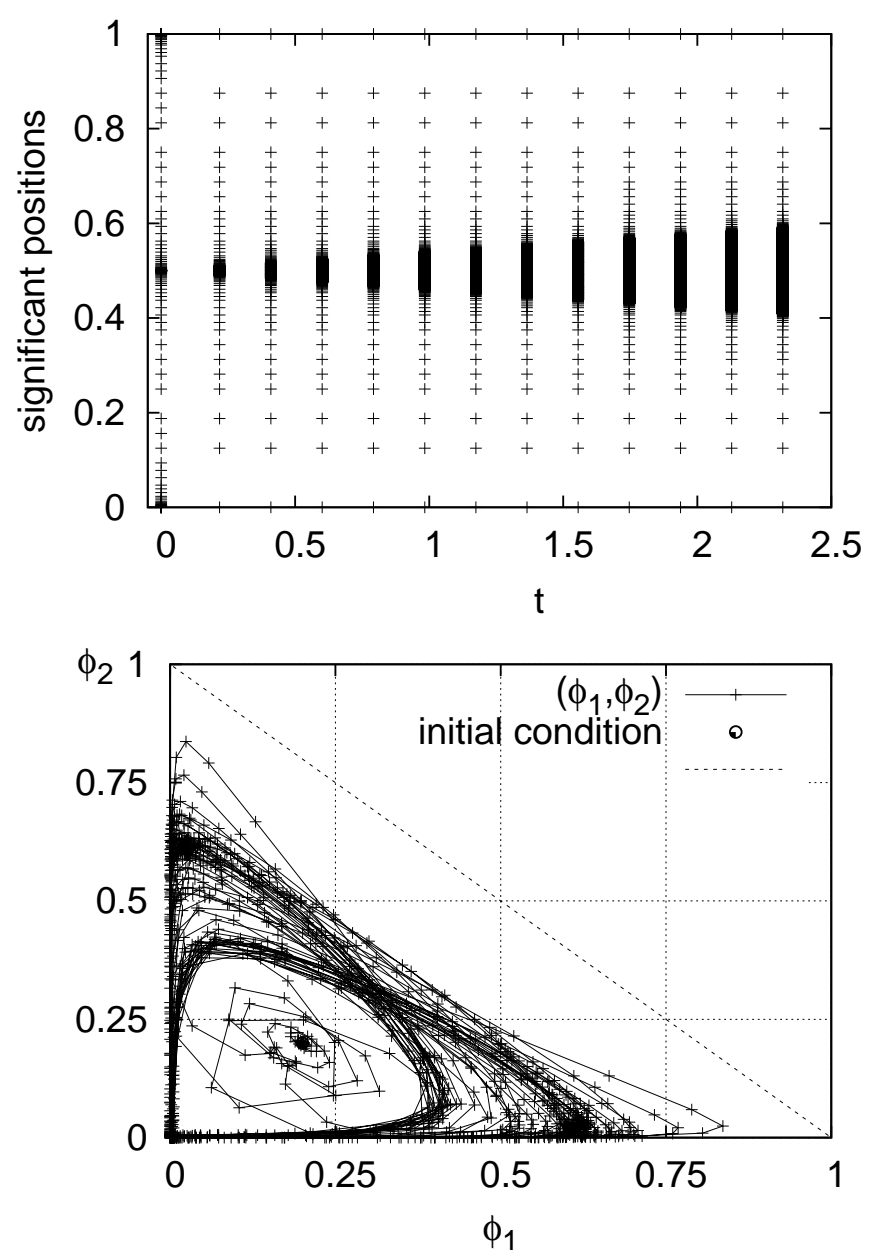

Figure 11: Example 4 (Model 2 with $\delta=1, \gamma=-1$ and $n=8$ ). Top: evolution of the SPR. Bottom: phase space at $t=1.74$.

We now solve the equation

$$
\tilde{\Delta}\left(1-\phi_{2}, \phi_{2}\right)=0 \text {, }
$$

to obtain candidates for tangent points. For $\delta=1$, we obtain $\phi_{1}=\phi_{2}=1 / 2$. For $\delta \neq 1$, the unique positive solutions of this equation are

$$
\phi_{2}=r_{3}(n, \delta) \pm \sqrt{\frac{\delta}{n(1-\delta)}+\left(r_{3}(n, \delta)\right)^{2}}, \quad r_{3}(n, \delta):=-\frac{1+\delta}{2 n}\left(\frac{1}{1-\delta}-n\right) .
$$

The quantity $r_{3}(n, \delta)$ may have either sign, but for $\delta \neq 1,(4.14)$ has exactly one positive solution $\phi_{2}=\phi_{2}^{*}$. We have $\phi_{2}^{*} \in(0,1]$ if and only if

$$
n \delta(1-\delta) \leqslant 1 .
$$


For convenience, we define $\phi_{2}^{*}=1 / 2$ when $\delta=1$. In that case, $\phi_{2}^{*}$ is a tangent since it is the intersection of a minima manifold with the antidiagonal, as shown in the sequel for a representative special case.

So far we have focused on providing exact algebraic expressions for the points at which $\partial \mathcal{E}_{2}(n, \gamma, \delta)$ is tangent to the boundary of $\mathcal{D}_{1}$. Of course, it is still necessary to show that $\partial \mathcal{E}_{2}(n, \gamma, \delta)$ is a closed curve located within $\mathcal{D}_{1}$. Fig. 6 suggests that this is indeed the case. Of course, this observation cannot replace a rigorous proof; but, on the other hand, we do not attempt here to provide a full characterization by means of analysis, but rather construct some interesting cases for a numerical simulation. A discussion of the shape of the curve $\partial \mathcal{E}_{2}(n, \gamma, \delta)$ will be limited to the "symmetric heavy-buoyant" case $\delta=1, \gamma=-1$.

\subsection{Symmetric case $\delta=1, \gamma=-1$}

Now we concentrate on the symmetric case where $\delta=1, \gamma=-1$. On the axes $\phi_{1}=0$ and $\phi_{2}=0$ there are tangents at the solutions of (4.10) and (4.11). Noting that

$$
r_{1}(n,-1,1)=r_{2}(n,-1,1)=n^{2}-8 n,
$$

we obtain

$$
\phi_{1}=\phi_{2}=\frac{1}{2} \pm \frac{\sqrt{n^{2}-8 n}}{2 n}
$$

where the number of solutions depends on the existence of real roots. This means that for $n=8, \partial_{2} \mathcal{E}(n,-1,1)$ has one single tangent to each axis, which are located at $\phi_{1}=1 / 2$ and $\phi_{2}=1 / 2$. For $n<8$ there are no tangents to the axes, and for $n>8$ there are two tangents to each axis that are moving towards the values 0 and 1 in the limit $n \rightarrow \infty$.

Now it is shown that there is a unique global minimum of the discriminant, which implies that the elliptic region is connected. On the diagonals $\phi_{1}=\phi-\phi_{2}$ (where $\phi$ is fixed) for $\delta=1$ and $\gamma=-1$ the discriminant becomes

$$
\begin{aligned}
\Delta\left(\phi-\phi_{2}, \phi_{2}\right)= & (1-\phi)^{2 n-4}\left(n^{2} \phi^{4}-4 n^{2} \phi_{2} \phi^{3}-2 n^{2} \phi^{3}+8 n^{2} \phi_{2} \phi^{2}\right. \\
& +4 n^{2} \phi_{2}^{2} \phi^{2}+n^{2} \phi^{2}+4 n \phi^{2}-8 n^{2} \phi_{2}^{2} \phi-16 \phi_{2} \phi \\
& \left.-4 n^{2} \phi_{2} \phi-4 n \phi+4+16 \phi_{2}^{2}+4 n^{2} \phi_{2}^{2}\right) .
\end{aligned}
$$

The expression $\Delta\left(\phi-\phi_{2}, \phi_{2}\right)$ assumes its unique minimum (for $\left.\phi \neq 1\right)$ at $\phi_{2}=\phi / 2$. In turn, on this minimum line $\phi_{1}=\phi_{2}$, the discriminant reads

$$
\Delta(\phi / 2, \phi / 2)=4 \mu^{2}(1-\phi)^{2 n-3}(1+(1-n) \phi), \quad(\text { for } \gamma=-1, \delta=1) .
$$

This expression has a local minimum at

$$
\phi=\frac{3 n-4}{2(n-1)^{2}}
$$



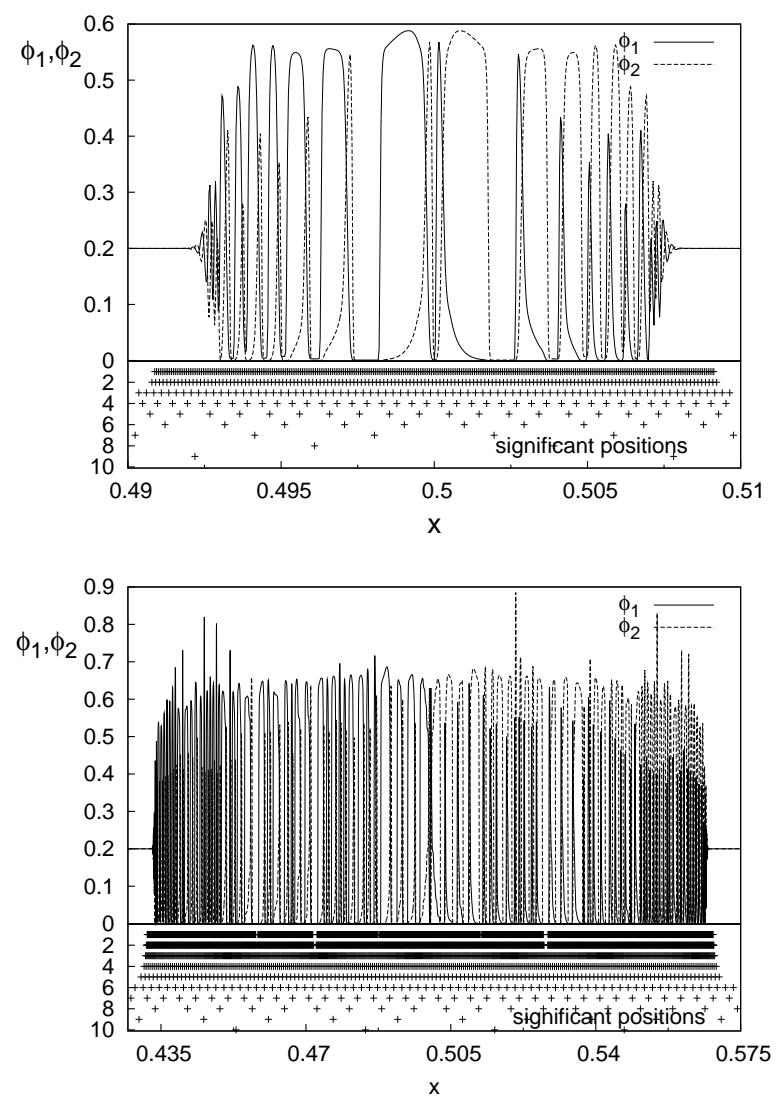

Figure 12: Example 4 (Model 2 with $\delta=1, \gamma=-1$ and $n=8$ ): profiles of $\phi_{1}$ and $\phi_{2}$ with significant positions at $t=0.21$ (top) and $t=1.74$ (bottom).

and its maximum at $\phi=1$. Thus, the global minimum of the discriminant has been found. This implies the connectedness of level sets with given levels, in particular that with vanishing discriminant.

\subsection{Simulation of Model 2}

In the numerical simulations for Model 2 (Examples 3 to 6), we illustrate how the oscillatory pattern depends on the shape, location, and points of tangency of the elliptic region $\mathcal{E}_{2}=\mathcal{E}_{2}(n, \gamma, \delta)$ with respect to $\mathcal{D}_{\phi_{\max }}$.

In all examples, the initial concentrations are constants that are disturbed at only three grid cells. The perturbation is then introduced at the middle point and at its two immediate neighbors of the discretization of the initial condition. This middle point is in fact the corresponding middle value of the vector of positions of the finest grid. Numerical tests show that the speed how fast the spiral develop depends on the strength of perturbation, i.e., with a minor degree of perturbation it takes longer to obtain the same number of peaks. However, the magnitude of small perturbations 

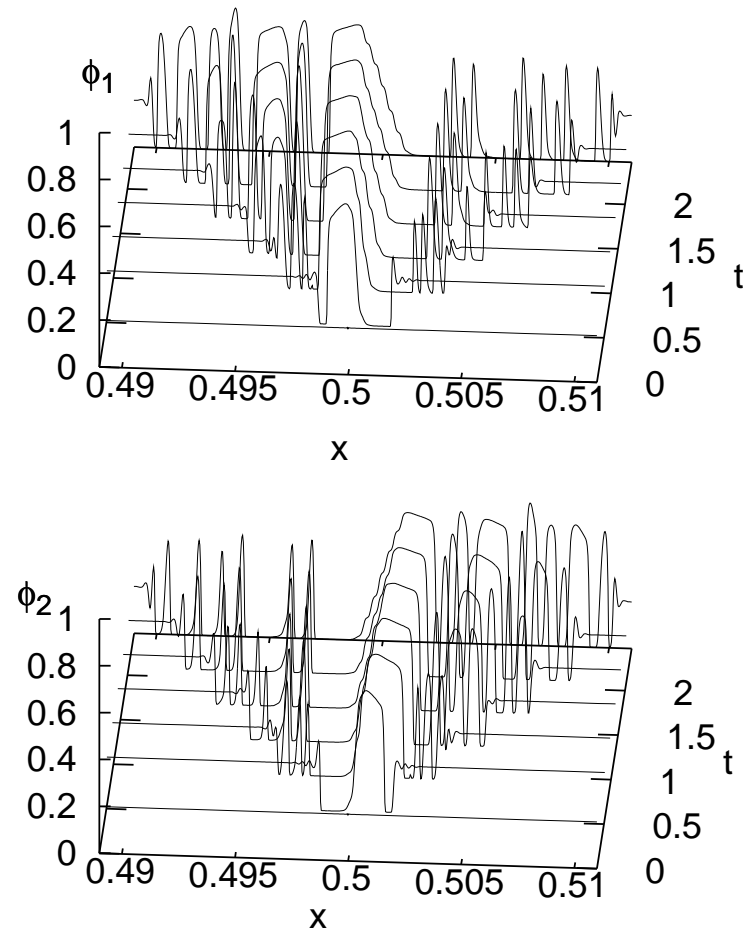

Figure 13: Example 5 (Model 2 with $\delta=1, \gamma=-1, n=12$ ): profiles of $\phi_{1}$ (top) and $\phi_{2}$ (bottom) at several times.

does not affect the shape of the oscillations.

In Section 3.2, it has already been recognized for Model 1 that the grid precision eventually controls the number of oscillations. Therefore, for our numerical simulations, the finest resolution level necessary to define the discretization of the initial condition contains $N_{0}=2^{15}$ points. This parameter has been adapted such that the examples are sufficiently fine to identify the desired effects, but still coarse enough so that individual peaks of oscillations are visible. For a vector

$$
\Phi_{0}=\left(\phi_{1,0}, \phi_{2,0}\right)^{\mathrm{T}} \in \mathcal{E}_{2}(n, \gamma, \delta),
$$

we then start from (2.5). These initial fine-grid values are specified as follows, where $i_{*}$ is the vector index corresponding to the middle point $x_{\mathrm{m}}$ of the domain:

$$
\phi_{i, 0}\left(x_{j}^{0}\right)= \begin{cases}\phi_{i, 0}, & \text { if } j<i_{*}-1 \text { or } j>i_{*}+1, \\ \phi_{i, 0}+0.002, & \text { if } j=i_{*}-1 \text { or } j=i_{*}+1, \quad i=1,2, \quad j=0, \ldots, N_{0} . \\ \phi_{i, 0}-0.004, & \text { if } j=i_{*},\end{cases}
$$

In Examples 3 to 6 we set $\delta=1, \bar{\varrho}_{1}=50, \bar{\varrho}_{2} \in\{-50,0\}$ for the parameter choices $\gamma \in\{-1,0\}$, $\Phi_{0}=(0.2,0.2), n \in\{4,4.65,8,12\}, x_{\mathrm{m}}=0.5, T=2.5$, and $\phi_{\max }=1.0$. 

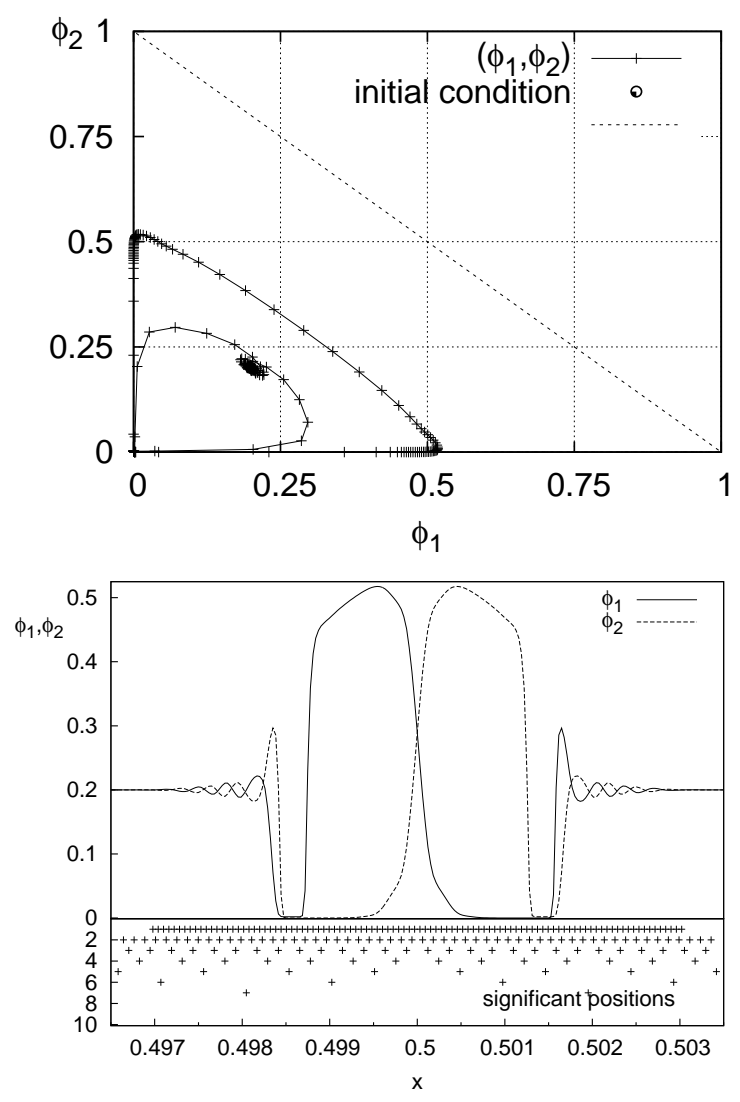

Figure 14: Example 5 (Model 2 with $\delta=1, \gamma=-1, n=12$ ). Top: phase space. Bottom: profiles of $\phi_{1}$ and $\phi_{2}$ with significant positions at time $t=0.55$.

\subsubsection{Examples 3, 4 and 5: Model 2 with $n \in\{4,8,12\}$, heavy-buoyant particles and almost constant initial data}

We now consider the parameters $\delta=1$ and $\gamma=-1$, corresponding to a heavy-buoyant bidisperse suspension of equal-sized spheres, and the exponents $n=4, n=8$, and $n=12$ in Examples 3, 4, and 5, respectively. In all numerical simulations the CFL number is set to 1.5 , and the threshold value is $\varepsilon_{0}=5 \times 10^{-8}$. In Fig. 8 the case $n=4$ is illustrated, where no oscillations occur. Comparing the top and bottom plots it can be seen that the profile of $\phi_{1}$ is symmetric to that of $\phi_{2}$, and both profiles form a similarity solution, i.e., depend on $\left(x-x_{\mathrm{m}}\right) / t$ only. The almost symmetry of the phase space plot (top plot of Fig. 9) with respect to the diagonal $\phi_{1}=\phi_{2}$ confirms this observation of symmetry. Finally, the number of grid points increases as $t$ increases, but as the bottom plot of Fig. 9 illustrates, the finest levels of multiresolution are occupied only near where the solution is discontinuous.

In Figs. $10-12$ and 13-15 the corresponding solutions for the respective cases $n=8$ and $n=12$ are presented. These figures indicate a strong connection between the shape, location and tangency properties of the elliptic region $\mathcal{E}_{2}$ inside $\mathcal{D}_{\phi_{\max }}$ and 

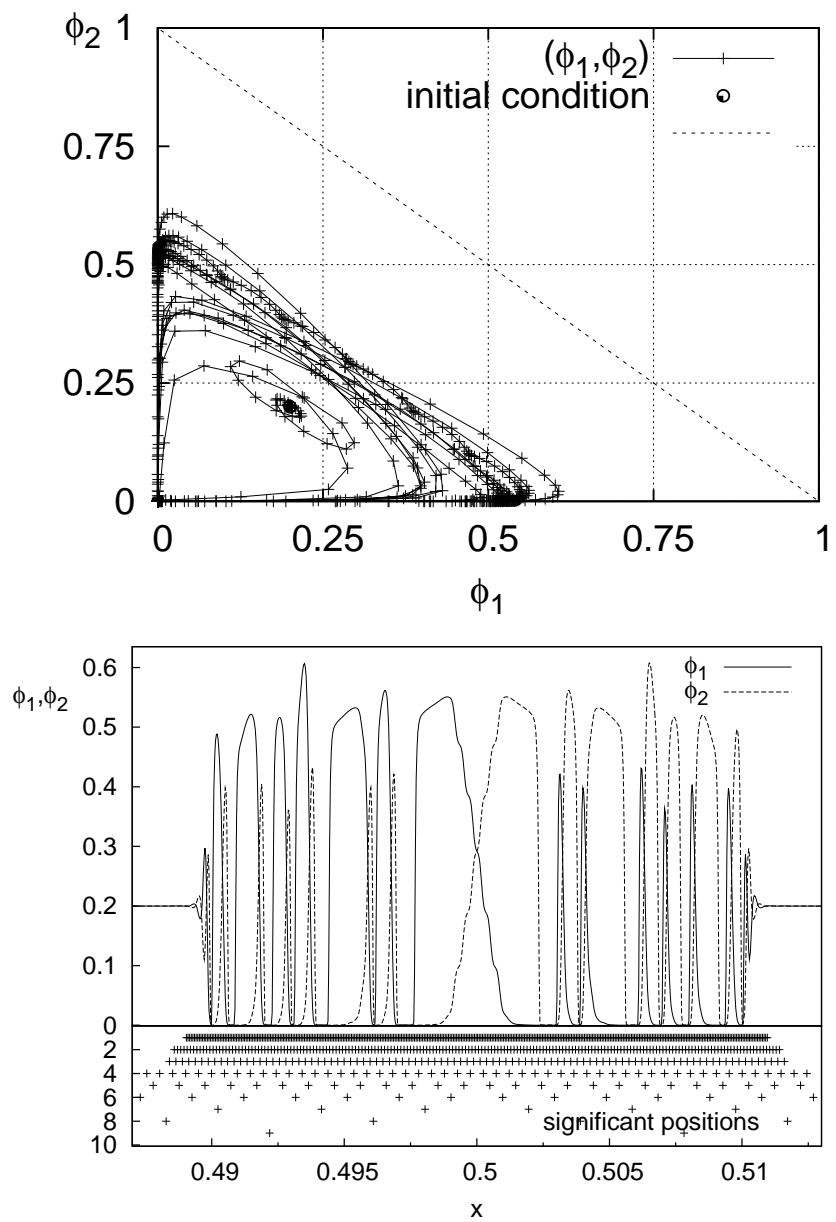

Figure 15: Example 5 (Model 2 with $\delta=1, \gamma=-1, n=12$ ). Top: phase space. Bottom: profiles of $\phi_{1}$ and $\phi_{2}$ with significant positions at time $t=2.47$.

the potential of oscillations. In the case $n=4$, the elliptic region $\mathcal{E}_{2}(4,-1,1)$ touches the boundary of $\mathcal{D}_{1}$ region only once on the antidiagonal $\phi_{1}+\phi_{2}=1$, leaving the axes $\phi_{1}=0$ and $\phi_{2}=0$ untouched (see Fig. 7). Thus, for large times it is expected that all oscillations disappear. However, for small times, oscillations are generated while the solution is still contained by the elliptic region. The elliptic region $\mathcal{E}_{2}(8,-1,1)$ touches each side of $\mathcal{D}_{1}$. For $n=12$, the elliptic region $\mathcal{E}_{2}(12,-1,1)$ touches line $\phi_{1}+\phi_{2}=1$ once and each of the axes $\phi_{1}=0$ and $\phi_{2}=0$ exactly twice, see Fig. 6 .

\subsubsection{Example 6: Model 2 with $n=4.65$, heavy-neutrally buoyant particles and al- most constant initial data}

The elliptic region $\mathcal{E}_{2}(4.65,0,1)$ touches the $\phi_{1}$-axis twice and stays away from the $\phi_{2}-$ axis. Figs. 16 and 17 show the corresponding numerical results, which are discussed in Section 5 in the light of experimental findings. 

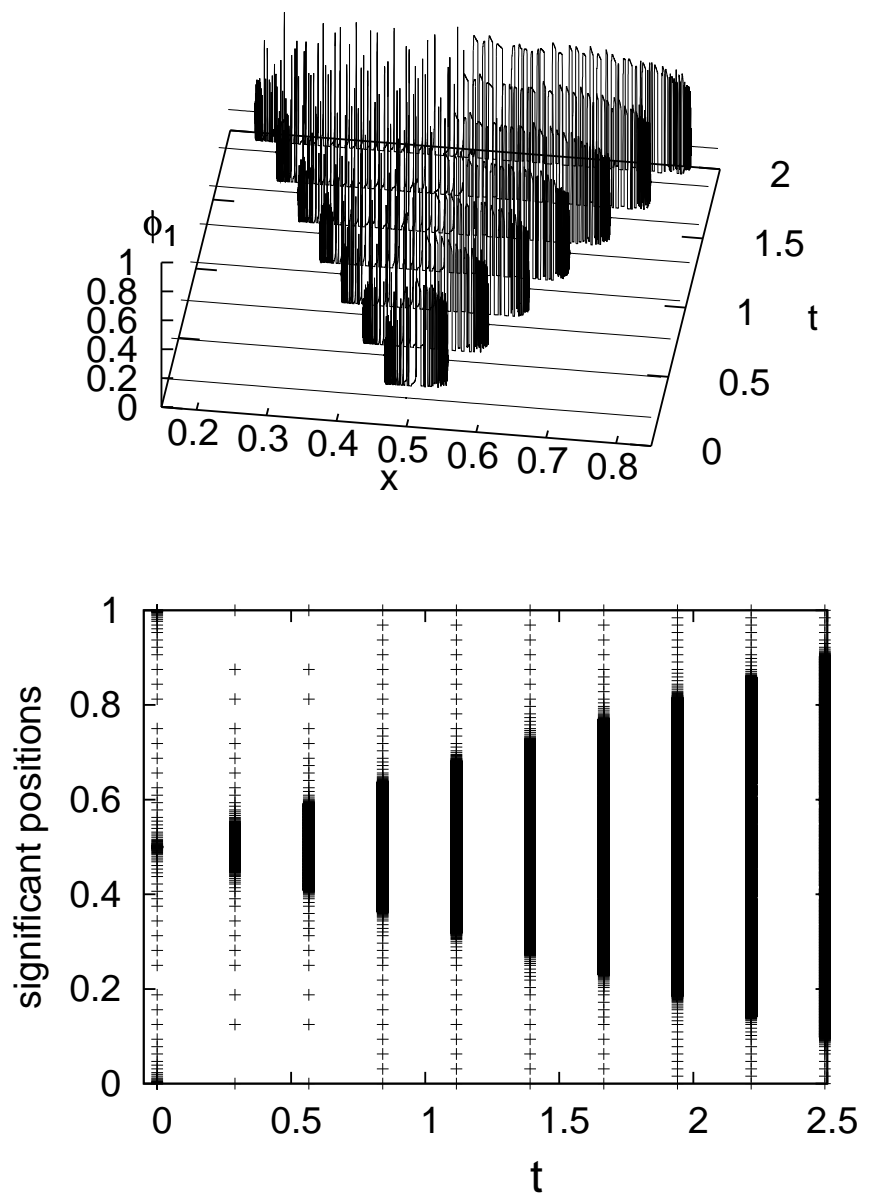

Figure 16: Example 6 (Model 2 with $\delta=1, \gamma=0, n=4.65$ ). Top: profiles of $\phi_{1}$ at several times. Bottom: evolution of the set of significant positions.

\section{Conclusions}

To put the present paper into the proper context, let us mention that it had been known from [22] (see also [23,24]) that initial value problems for hyperbolic-elliptic systems develop strongly oscillating solutions when the initial datum is a slightly perturbed constant chosen from the interior of the elliptic region. The fact that for Model 1 the elliptic region $\mathcal{E}_{1}$ is tangent to each side of the invariant region $\nabla$ was in the first place not considered crucial. In the beginning, the authors conjectured that similar oscillations would occur in the model of polydisperse sedimentation for particles with different densities (Model 2) under standard choices of parameters (in particular, $n \approx 5$ ). However, we observed and reported some anomalous phenomena such as mutual blocking of buoyant and heavy particles (see [5, Fig. 10]; that situation is comparable with our Example 3 (Fig. 8)), but oscillations were not observed. The absence of oscil- 

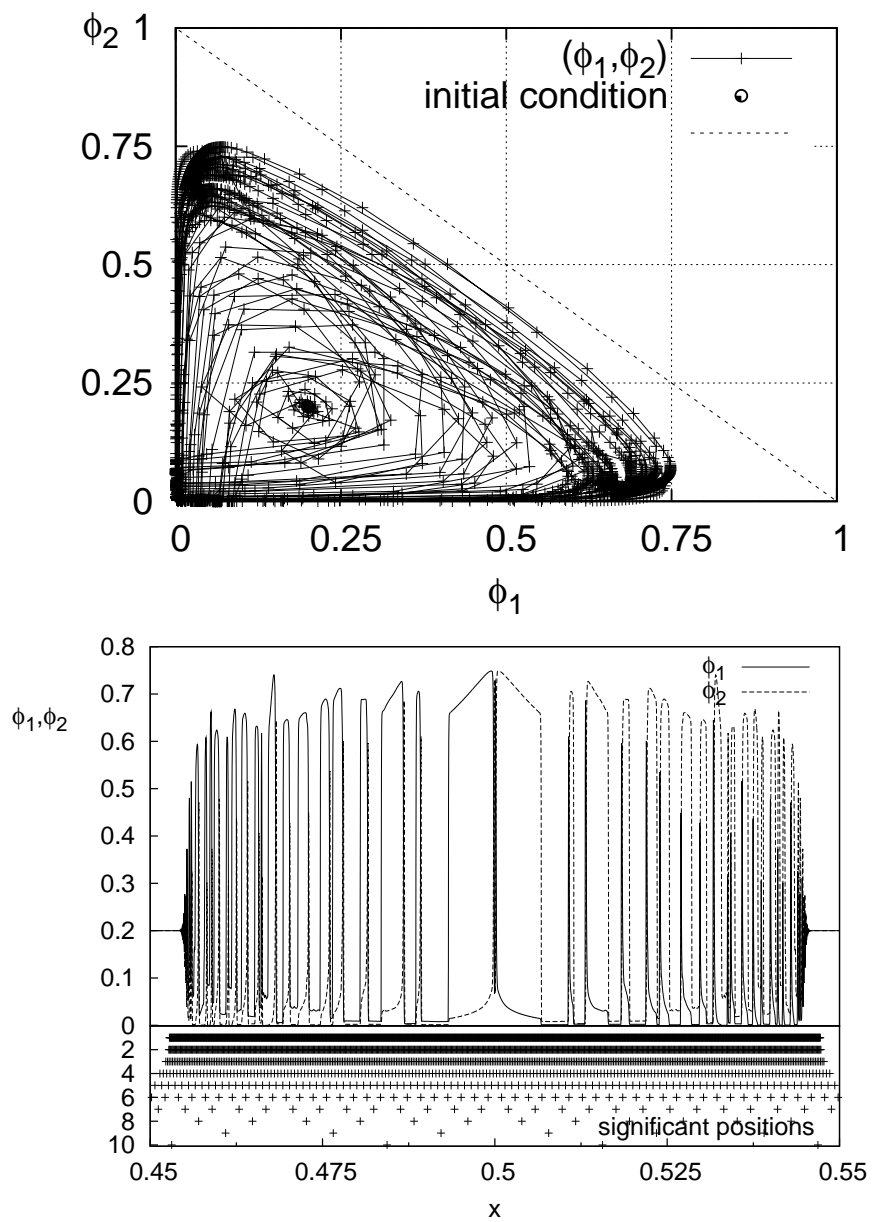

Figure 17: Example 6 (Model 2 with $\delta=1, \gamma=0, n=4.65$ ). Top: phase space. Bottom: profiles of $\phi_{1}$ and $\phi_{2}$ with significant positions at time $t=0.29$.

lations was first attributed to a wrong choice of initial data or a strong numerical viscosity of the scheme. Only after ruling out several possible reasons we realized that the shape, location, and number of points of tangency of the elliptic region $\mathcal{E}_{2}=\mathcal{E}_{2}(n, \gamma, \delta)$ with respect to the phase space $\mathcal{D}_{\phi_{\max }}$ is decisive for the appearance of oscillations. As the discussion of Section 4.3 shows, for the case of our Model 2, cases with more than one point of tangency, and which do give rise to oscillations, can be produced if the exponent $n$ assumes sufficiently large values. In fact, for Model 2 it seems that only in the case when each side of the triangular phase space $\mathcal{D}_{\phi_{\max }}$ is touched by the boundary of $\mathcal{E}_{2}$ we may expect oscillations to occur. A situation where $\mathcal{E}_{2}$ comes close to the boundary of $\mathcal{D}_{\phi_{\max }}$, but does not touch it, seems to cause oscillations to fade out as soon as solution values exit the elliptic region.

Let us briefly comment on our use of the MR method to capture oscillatory waves. The basic hypothesis is that adaptivity is needed whenever an "oscillation wave" oc- 
curs. The concept of "oscillation wave" is coined in analogy to rarefaction or shock waves [22]. The necessity of refinement at an oscillation wave can be quantified by the compression rate, as is illustrated in Fig. 3. However, by refining the finest possible grid (i.e., replacing, for example, $N_{0}$ by $2 N_{0}$ ), Fig. 4 suggests nothing like convergence in the classical sense. Rather, when the cell width is halved then also the period of an oscillatory cycle is halved. Note that the shape (but not the period) of a sinusoidal wave is retained and probably also some statistical average properties.

Finally, let us point out that although Model 2 is spatially one-dimensional, it still provides information about when the instability phenomena described in Section 4.2, which are based on an at least two-dimensional model concept, are likely to occur. This due to the fact that the multi-dimensional version of (4.4) is given by the equation

$$
\partial_{t} \Phi+\nabla \cdot(\Phi \otimes \boldsymbol{q}+\boldsymbol{f}(\Phi) \otimes \boldsymbol{k})=0
$$

where $q$ is the volume-averaged mixture flow velocity and $k$ is the upwards-pointing unit vector, and that for a given vector $\Phi$, the type of this equation coincides with that of (4.4). For the case of bidisperse suspensions with $\delta \approx 1$ and $\gamma \approx-1$, the theoretical prediction of the onset of instabilities was found to be in good agreement with experimental observations by [2]; that analysis, in turn, was based on experimental data by Weiland et al. [50] (see also [18]), and is mainly based on heavy-buoyant systems with $\delta \approx 1, \gamma \approx-1$; these values correspond to our (hypothetical) Examples 3, 4 and 5. More recently, Liu et al. [37] conducted a particle-scale computational analysis of the same phenomenon and observed instabilities. On the other hand, experimental observations of bidisperse suspensions with heavy and neutrally buoyant particles, as considered in our Example 6, go back to as early as Whitmore's classical paper [51] published in 1955. At current it is not yet clear whether numerical results such as those of Example 6 (Figs. 16 and 17), which were obtained for the realistic exponent $n=4.65$, have a direct physical meaning or rather alert to some instability phenomenon that could be captured by a multi-dimensional simulation only. Nevertheless, it is tempting to interpret the peaks forming in the concentration profiles of Figs. 16 and 17 as the formation of particle clusters, which would be consistent with Whitmore's observation concerning the structure of vertical currents ("streams") in the sedimenting system: "When the total concentration [ $\phi$ in our terminology] exceeded 30-35\%, the streams became increasingly granular in appearance as though particles were forming into loose conglomerates while falling" [51, p. 244].

\section{Acknowlegments}

SB acknowledges support by Conicyt (Chile) through Fondecyt project 11080253. RB acknowledges support by Conicyt (Chile) through Fondecyt project 1090456, Fondap in Applied Mathematics, project 15000001, and BASAL project CMM, Universidad de Chile and Centro de Investigación en Ingeniería Matemática $\left(\mathrm{CI}^{2} \mathrm{MA}\right)$, Universidad de Concepción. AK is supported by CNPq project No. 476022/2007-0. 


\section{References}

[1] A.V. Azevedo, D. Marchesin, B. Plohr and K. Zumbrun, Capillary instability in models for three-phase flow, Z. Angew. Math. Phys., 53 (2002), pp. 713-746.

[2] G.K. BATCHELOR AND R.W. JANSE VAN RENSBURG, Sedimentation in a dilute polydisperse system of interacting spheres, J. Fluid Mech., 119 (1982), pp. 379-408.

[3] J.B. BeLL, J.A. TRANGENSTEIN AND G.R. SHuBIN, Conservation laws of mixed type describing three-phase flows in porous media, SIAM J. Appl. Math., 46 (1986), pp. 1000-1017.

[4] S. BERRES AND R. BÜRGER, On the settling of a bidisperse suspension with particles having different sizes and densities, Acta Mech., 201 (2008), pp. 47-62.

[5] S. BERRES, R. BÜRGER AND K.H. KARLSEN, Central schemes and systems of conservation laws with discontinuous coefficients modeling gravity separation of polydisperse suspensions, J. Comput. Appl. Math., 164-165 (2004), pp. 53-80.

[6] S. BERRES, R. BÜRGER, K.H. KARLSEN AND E.M. TORY, Strongly degenerate parabolichyperbolic systems modeling polydisperse sedimentation with compression, SIAM J. Appl. Math., 64 (2003), pp. 41-80.

[7] P.M. Biesheuvel, H. VerWeij AND V. BReEdveld, Evaluation of instability criterion for bidisperse sedimentation, AIChE J., 47 (2001), pp. 45-52.

[8] R. BÜRGER, F. CONCHA, K.-K. FJELDE AND K.H. KARLSEN, Numerical simulation of the settling of polydisperse suspensions of spheres, Powder Technol., 113 (2000), pp. 30-54.

[9] R. BÜRGER, K.-K. FJELDE, K. HÖFLER AND K.H. KARLSEN, Central difference solutions of the kinematic model of settling of polydisperse suspensions and three-dimensional particle-scale simulations, J. Eng. Math., 41 (2001), pp. 167-187.

[10] R. BÜRGER, A. GARCÍA AND M. KUNIK, A generalized kinetic model of sedimentation of polydisperse suspensions with a continuous particle size distribution, Math. Models Methods Appl. Sci., 18 (2008), pp.1741-1785.

[11] R. BÜRGER, K.H. KARLSEN, E.M. TORY AND W.L. WENDLAND, Model equations and instability regions for the sedimentation of polydisperse suspensions of spheres, ZAMM Z. Angew. Math. Mech., 82 (2002), pp. 699-722.

[12] R. BÜRGER AND A. KOZAKEVICIUS, Adaptive multiresolution WENO schemes for multispecies kinematic flow models, J. Comput. Phys., 224 (2007), pp. 1190-1222.

[13] R. BÜrger, A. KozAKeviciUs AND M. SEpÚlvedA, Multiresolution schemes for strongly degenerate parabolic equations, Numer. Meth. Partial Diff. Eqns., 23 (2007), pp. 706-730.

[14] G. ChiAVASSA AND R. DONAT, Point value multiscale algorithms for 2D compressive flows, SIAM J. Sci. Comput., 23 (2001), pp. 805-823.

[15] G. Chiavassa, R. DONAT AND S. MÜLler, Multiresolution-based adaptive schemes for hyperbolic conservation laws. In: T. PleWA, T. LINDE AND V.G. WEISS (EDS.), Adaptive Mesh Refinement-Theory and Applications, Lecture Notes in Computational Science and Engineering vol. 41, Springer-Verlag, Berlin (2003), pp. 137-159.

[16] R.H. DAVIS, Hydrodynamic diffusion of suspended particles: a symposium, J. Fluid Mech., 310 (1996), pp. 325-335.

[17] D.L. DONOHO, Interpolating wavelet transforms, Technical Report 408, Department of Statistics, Stanford University, Nov. 1992.

[18] Y.P. FESSAS AND R.H. WEILAND, The settling of suspensions promoted by rigid buoyant particles, Int. J. Multiphase Flow, 10 (1984), pp. 485-507.

[19] A.D. FITT, The numerical and analytical solution of ill-posed systems of conservation laws, Appl. Math. Modelling, 13 (1989), pp. 618-631.

[20] A.D. FITT, Mixed systems of conservation laws in industrial mathematical modelling, Surv. 
S. Berres, R. Bürger, A. Kozakevicius / Adv. Appl. Math. Mech., 5 (2009), pp. 581-614

Math. Indust., 6 (1996), pp. 21-53.

[21] H. FRID, Existence and asymptotic behavior of measure-valued solutions for three-phase flow in porous media, J. Math. Anal. Appl., 196 (1995), pp. 614-627.

[22] H. FRID AND I-S. LIU, Oscillation waves in Riemann problems inside elliptic regions for conservation laws of mixed type, Z. Angew. Math. Phys., 46 (1995), pp. 913-931.

[23] H. FRID AND I-S. LIU, Phase transitions and oscillation waves in an elastic bar, Mat. Contemp., 10 (1996), pp. 123-135.

[24] H. FRID AND I-S. LIU, Oscillation waves in Riemann problems for phase transitions, Quart. Appl. Math., 56 (1998), pp. 115-135.

[25] A. HARTEN, Multiresolution algorithms for the numerical solution of hyperbolic conservation laws, Comm. Pure Appl. Math., 48 (1995), pp. 1305-1342.

[26] A. HARTEN, Multiresolution representation of data: a general framework, SIAM J. Numer. Anal., 33 (1996), pp. 1205-1256.

[27] H. Holden, L. Holden AND N.H. RiseBRO, Some qualitative properties of $2 \times 2$ systems of conservation laws of mixed type. In: B.L. KEYFITZ AND M. SHEARER (EDS.), Nonlinear Evolution Equations that Change Type. IMA Vol. in Math. and Its Appl. vol. 27, SpringerVerlag, New York (1990), pp. 67-78.

[28] M. HOLMSTRÖM, Solving hyperbolic PDEs using interpolating wavelets, SIAM J. Sci. Comput., 21 (1999), pp. 405-420.

[29] L. JAMESON, A wavelet-optimized, very high order adaptive grid and order numerical method, SIAM J. Sci. Comput., 19 (1998), pp. 1980-2013.

[30] B.L. KEYFITZ, A geometric theory of conservation laws which change type, ZAMM Z. Angew. Math. Mech., 75 (1995), pp. 571-581.

[31] B.L. KEYFITZ, Mathematical properties of nonhyperbolic models for incompressible two-phase flow. In: Proceedings of the International Conference on Multiphase Flow, New Orleans, May 27-June 1, 2001 (CD-ROM).

[32] A.J. KOZAKEVICIUS AND L.C.C. SANTOS, ENO adaptive method for solving one-dimensional conservation laws, Appl. Numer. Math., 59 (2009), pp. 2337-2355.

[33] A. KURGANOV AND E. TADMOR, New high resolution central schemes for nonlinear conservation laws and convection-diffusion equations, J. Comput. Phys., 160 (2000), pp. 241-282.

[34] A.J.C. LADD, Dynamical simulations of sedimenting spheres, Phys. Fluids, 5 (1993), pp. 299 310.

[35] H.-S. LAW, J.H. MasliYah, R.S. MacTagGaRT AND K. NANDAKUMAR, Gravity separation of bidisperse suspensions: light and heavy particle species, Chem. Eng. Sci., 42 (1987), pp. 1527-1538.

[36] R.J. Le Veque, Finite Volume Methods for Hyperbolic Problems, Cambridge University Press, Cambridge, UK, 2002.

[37] X. LIU, W. GE AND J. LI, Non-equilibrium phase transitions in suspensions of oppositely driven inertial particles, Powder Technol. 184 (2008), pp. 224-231.

[38] M.J. LOCKETT AND K.S. BASSOON, Sedimentation of binary particle mixtures, Powder Technol., 24 (1979), pp. 1-7.

[39] A. MAJDA AND R.L. Pego, Stable viscosity matrices for system of conservation laws, J. Differential Equations, 56 (1985), pp. 229-262.

[40] D. MARCHESIN AND B. PlOHR, Wave structure in WAG recovery. SPE paper 56480.

[41] D. MARCHESIN AND B. PLOHR, Theory of three-phase flow applied to water-alternating-gas enhanced oil recovery. In: H. FREISTÜHLER AND G. WARNECKE (EDS.), Hyperbolic Problems: Theory, Numerics, Applications, Vol. II, Birkhäuser Verlag, Basel (2001), pp. 693702. 
[42] J.H. MasliyaH, Hindered settling in a multiple-species particle system, Chem. Eng. Sci., 34 (1979), pp. 1166-1168.

[43] S. MÜLlER, Adaptive Multiscale Schemes for Conservation Laws, Springer-Verlag, Berlin, 2003.

[44] H. NESSYAHU AND E. TADMOR, Non-oscillatory central differencing for hyperbolic conservation laws, J. Comput. Phys., 87 (1990), pp. 408-463.

[45] R.L. PEgO AND D. SERRE, Instabilities in Glimm's scheme for two systems of mixed type, SIAM J. Numer. Anal., 25 (1988), pp. 965-988.

[46] C.-W. SHU, Essentially non-oscillatory and weighted essentially non-oscillatory schemes for hyperbolic conservation laws. In: B. COCKBURN, C. JOHNSON, C.-W. SHU AND E. TADMOR, Advanced Numerical Approximation of Nonlinear Hyperbolic Equations (A. Quarteroni, Ed.), Lecture Notes in Mathematics vol. 1697, Springer-Verlag, Berlin (1998), pp. 325-432.

[47] C.-W. SHU, High order Weighted Essentially Nonoscillatory schemes for convection dominated problems, SIAM Rev., 51 (2009), pp. 82-126.

[48] E.F. TORO, Riemann Solvers and Numerical Methods for Fluid Dynamics, Third Edition, Springer, Berlin, 2009.

[49] E.M. TORY, Stochastic sedimentation and hydrodynamic diffusion, Chem. Eng. J., 80 (2000), pp. 81-89.

[50] R.H. WEILAND, Y.P. FESSAS AND B.V. RAMARAO, On instabilities arising during sedimentation of two-component mixtures of solids, J. Fluid Mech., 142 (1984), pp. 383-389.

[51] R.L. WHitMORE, The sedimentation of suspensions of spheres, Brit. J. Appl. Phys., 6 (1955), pp. 239-245.

[52] P. ZhANG, R.-X. LiU, S.C. WONG AND S.-Q. DAI, Hyperbolicity and kinematic waves of a class of multi-population partial differential equations, Eur. J. Appl. Math., 17 (2006), pp. 171200. 